\title{
Combretastatin A4 phosphate induces rapid regression of tumor neovessels and growth through interference with vascular endothelial-cadherin signaling
}

\author{
Loïc Vincent, ${ }^{1}$ Pouneh Kermani, ${ }^{2}$ Lauren M. Young, ${ }^{1}$ Joseph Cheng, ${ }^{1}$ Fan Zhang, ${ }^{1}$ \\ Koji Shido, ${ }^{1}$ George Lam, ${ }^{1}$ Heidi Bompais-Vincent, ${ }^{3}$ Zhenping Zhu, ${ }^{4}$ \\ Daniel J. Hicklin, ${ }^{4}$ Peter Bohlen, ${ }^{4}$ David J. Chaplin, ${ }^{5}$ Chad May, ${ }^{4}$ and Shahin Rafiii
}

\begin{abstract}
1Department of Genetic Medicine and Division of Hematology and Oncology, Howard Hughes Medical Institute, 2Department of Medicine, Weill Medical College of Cornell University, New York, New York, USA. ${ }^{2}$ Laboratory of Developmental Hematopoiesis, Memorial Sloan Kettering Cancer Center, New York, New York, USA. ${ }^{4}$ mClone Systems Inc., New York, New York, USA. ${ }^{5}$ OXiGENE Inc., Waltham, Massachusetts, USA.
\end{abstract}

\begin{abstract}
The molecular and cellular pathways that support the maintenance and stability of tumor neovessels are not well defined. The efficacy of microtubule-disrupting agents, such as combretastatin A4 phosphate (CA4P), in inducing rapid regression of specific subsets of tumor neovessels has opened up new avenues of research to identify factors that support tumor neoangiogenesis. Herein, we show that CA4P selectively targeted endothelial cells, but not smooth muscle cells, and induced regression of unstable nascent tumor neovessels by rapidly disrupting the molecular engagement of the endothelial cell-specific junctional molecule vascular endothelial-cadherin (VE-cadherin) in vitro and in vivo in mice. CA4P increases endothelial cell permeability, while inhibiting endothelial cell migration and capillary tube formation predominantly through disruption of VE-cadherin/ $\beta$-catenin/Akt signaling pathway, thereby leading to rapid vascular collapse and tumor necrosis. Remarkably, stabilization of VE-cadherin signaling in endothelial cells with adenovirus $E 4$ gene or ensheathment with smooth muscle cells confers resistance to CA4P. CA4P synergizes with low and nontoxic doses of neutralizing mAbs to VE-cadherin by blocking assembly of neovessels, thereby inhibiting tumor growth. These data suggest that the microtubule-targeting agent CA4P selectively induces regression of unstable tumor neovessels, in part through disruption of VE-cadherin signaling. Combined treatment with anti-VE-cadherin agents in conjunction with microtubule-disrupting agents provides a novel synergistic strategy to selectively disrupt assembly and induce regression of nascent tumor neovessels, with minimal toxicity and without affecting normal stabilized vasculature.
\end{abstract}

\section{Introduction}

Stability and functional assembly of tumor neovessels are governed by collaboration of multiple organ-specific cellular and angiogenic factors. The profound selective efficacy and rapid mode of action of prototypical microtubule-disrupting agents, such as combretastatin A4 phosphate (CA4P), in regressing tumor vasculature suggest that tumor neovessels are maintained and stabilized by as-yet-unrecognized activated cellular and molecular factors.

Targeted genetic manipulation and antibody-mediated inhibition of angiogenic growth factors and their receptors in murine tumor models have resulted in identification of key angiogenic modulators that support tumor neoangiogenesis. Neutralizing mAbs to VEGF-A or one of its receptors, VEGFR-2, a tyrosine kinase that regulates endothelial integrity, induces regression of

Nonstandard abbreviations used: AdNullE4+ ${ }^{+}$, adenoviral E4 ${ }^{+}$; CA4P, combretastatin A4 phosphate; HUVEC, human umbilical vein endothelial cell; VE-cadherin, vascular endothelial-cadherin; ZO-1, zona occludens 1.

Conflict of interest: D.J. Chaplin is the Chief Scientific Officer of and a shareholder in OXiGENE Inc., which holds the rights to produce combretastatin A4 phosphate. D.J. Hicklin, Z. Zhu, and C. May are employees of ImClone Systems Inc. and hold stock or stock options in the company. P. Bohlen was employed by ImClone Systems Inc. at the time of this study.

Citation for this article: J. Clin. Invest. 115:2992-3006 (2005). doi:10.1172/JCI24586. unstable neovessels within the tumor, thereby normalizing tumor vasculature (1). However, it remains to be determined which angiogenic factors maintain the stability of these normalized vessels. Therefore, understanding the mechanism by which vascular targeting agents disrupt the integrity of tumor neovessels will provide a platform to identify the molecular pathways involved in normalization and stabilization of subsets of tumor neovessels.

One other essential angiogenic factor that collaborates with VEGFR-2 to support the assembly of tumor neovessels is the endothelial adhesion molecule known as vascular endothelialcadherin (VE-cadherin), which localizes exclusively at specialized cell-cell contact regions of endothelium (2). In fact, VE-cadherin is involved in various aspects of vascular biology related to angiogenesis, including endothelial cell migration, survival, contactinduced growth inhibition, vascular integrity, and, most notably, endothelial cell assembly into tubular structures (3-7). Targeting of VE-cadherin has been effective in inducing regression of the tumor vasculature. However, given the critical role of VE-cadherin in maintaining the integrity of endothelial cells, targeting VE-cadherin has resulted in significant vascular toxicity $(8,9)$. Therefore, selective targeting of VE-cadherin within the tumor neovessels or decreasing the requirement for using high doses of anti-VEcadherin agents will significantly diminish toxicity associated with inhibition of VE-cadherin. A recent discovery that distinct 

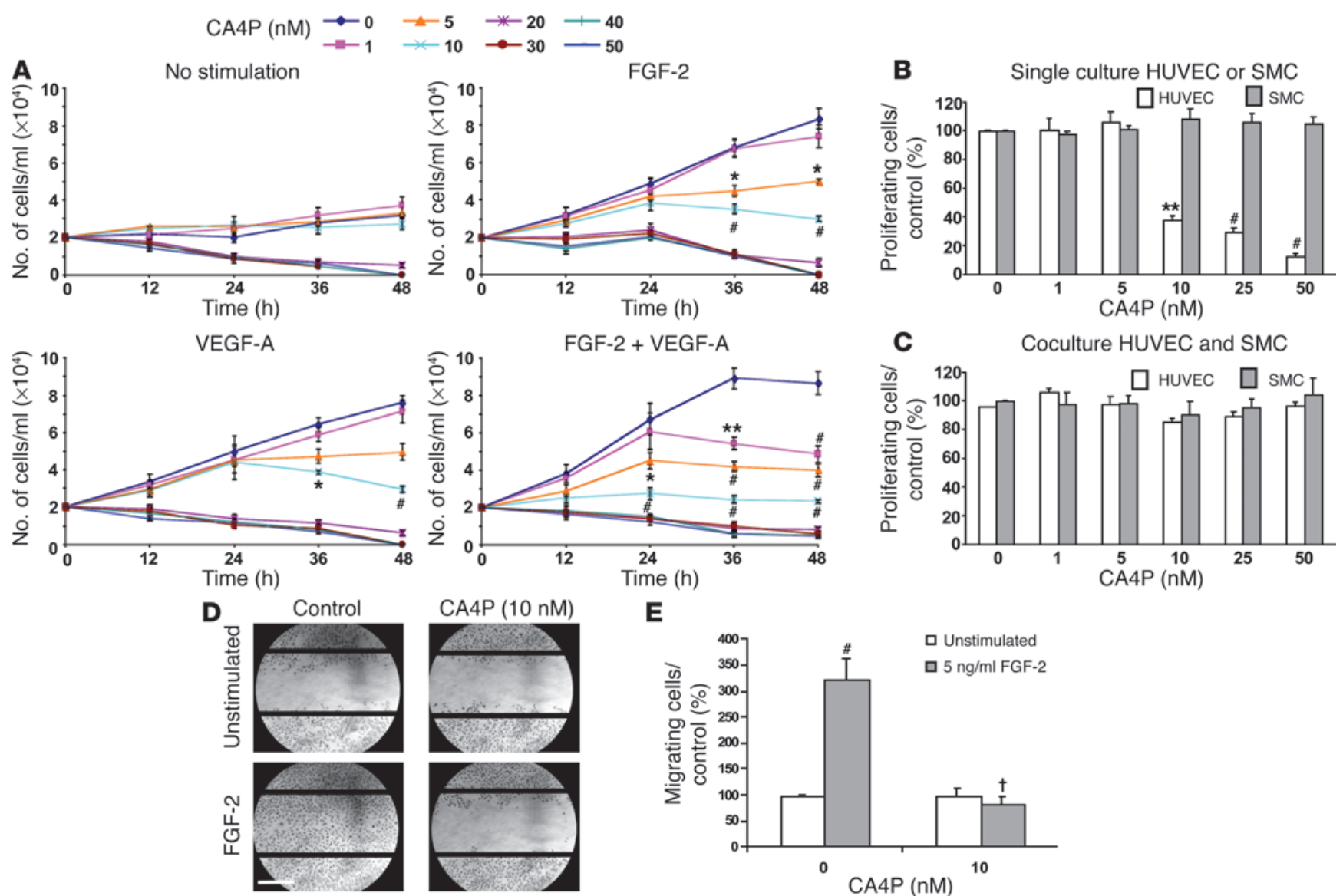

E

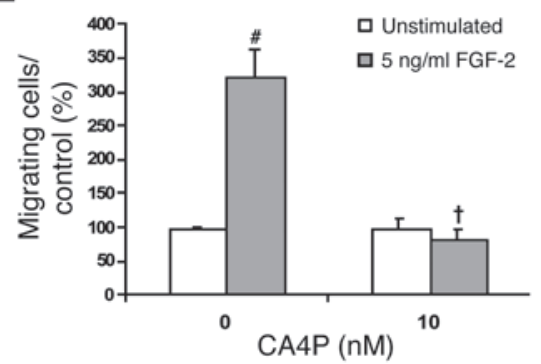

Figure 1

CA4P inhibits growth factor-induced endothelial cell proliferation and migration. (A) CA4P inhibits HUVEC proliferation. HUVECs were incubated with or without growth factors, and CA4P was added at different concentrations. Cells were then counted at the indicated time points. Results of 4 experiments in duplicate are expressed as the mean number of cells $\pm \mathrm{SEM}\left({ }^{\star} P<0.05,{ }^{\star \star} P<0.01,{ }^{\#} P<0.001\right.$ compared with CA4P-untreated cells; $n=4)$. (B) SMCs are not sensitive to CA4P. HUVECs or SMCs were incubated with CA4P, and cells were counted after 48 hours. Results of 4 experiments in duplicate are expressed as the mean number of cells \pm SEM $\left({ }^{* *} P<0.01,{ }^{\sharp} P<0.001\right.$ compared with CA4P-untreated cells; $\left.n=4\right)$. (C) HUVECs are resistant to CA4P when cocultured with SMCs. HUVECs and SMCs were seeded together, incubated with CA4P, and counted after 48 hours. Results of 4 experiments in duplicate are expressed as the mean number of cells \pm SEM. (D) CA4P inhibits HUVEC migration. A lesion was produced across the HUVECs' monolayers, and unstimulated or FGF-2-stimulated cell monolayers were incubated with CA4P for 24 hours and then photographed. A representative picture is shown. Magnification: $\times 4$. Scale bar, $500 \mu \mathrm{m}$. (E) Quantification of recovery of each denuded area after CA4P treatment. Results are expressed as the ratio of the number of invading cells to the number of migrating cells in absence of CA4P \pm SEM ( $\#<0.001$ compared with CA4P-untreated cells; ${ }^{\dagger} P<0.001$ compared with FGF-2-stimulated endothelial cells; $n=5$ ).

epitopes of VE-cadherin molecule are selectively expressed on neoangiogenic endothelial cells (9) will increase selective targeting of the tumor neovessels, while diminishing toxicity to normal organ vasculature. Nonetheless, identification of factors that can selectively enhance disengagement of VE-cadherin homodimers in tumor neovessels will increase the therapeutic efficacy and marginal safety of anti-VE-cadherin agents $(8,9)$.

Agents that can potentially influence VE-cadherin engagement are vascular targeting agents. Differences in the architecture, cellular, and biochemical composition between normal and tumor blood vessels provide the basis for design of vascular targeting of tumors. The newly formed tumor vessels are usually thin-walled capillaries or sinusoids with little more than an endothelial lining stabilized by a basement membrane and are prone to spontaneous hemorrhage and thrombosis (10-12). Dependence of tumor growth and progression on neoangiogenesis makes targeting of vasculature and inhibition of angiogenesis very important and promising anticancer strategies (13-15). Generally, in contrast to the antiproliferative effects of antiangiogenic therapy, antivascular approaches aim to cause rapid and catastrophic shutdown in the vascular function of the tumor, leading to extensive secondary tumor cell death (16-18). The antimitotic agent colchicine was among the first agents shown to possess antivascular activity, producing hemorrhagic necrosis in experimental tumors. Tubulin is also the site of action of the clinically used class of anticancer compounds, such as vinca alkaloids. These compounds exert their anticancer effect by binding to tubulin and preventing its polymerization to form microtubules, thus inhibiting a number of cellular processes, including mitosis (19).

Much interest has recently been generated in a series of stilbene compounds isolated from the South African tree Combretum caffrum. Combretastatin A4 has been shown to bind to tubulin at 
Table 1

CA4P does not induce endothelial cell apoptosis

$\begin{array}{llccc}\begin{array}{c}\text { CA4P } \\ \text { (nM) }\end{array} & \begin{array}{c}\text { Live } \\ \text { cells (\%) } \\ \text { AnnV-/PI- }\end{array} & \begin{array}{c}\text { Early apoptotic } \\ \text { cells (\%) } \\ \text { AnnV+/PI- }\end{array} & \begin{array}{c}\text { Late apoptotic } \\ \text { cells (\%) } \\ \text { AnnV+/PI+ }\end{array} & \begin{array}{c}\text { Dead } \\ \text { cells (\%) } \\ \text { AnnV-/PI+ }\end{array} \\ 0 & 98.0 \pm 1.2 & 0.5 \pm 0.2 & 0.8 \pm 0.4 & 0.7 \pm 0.1 \\ 10 & 57.8 \pm 5.4^{\mathrm{A}} & 2.2 \pm 0.8(\mathrm{NS}) & 7.4 \pm 1.3(\mathrm{NS}) & 32.6 \pm 3.3^{\mathrm{A}} \\ 50 & 33.3 \pm 4.5^{\mathrm{A}} & 3.7 \pm 1.1 \text { (NS) } & 9.9 \pm 1.8 \text { (NS) } & 53.1 \pm 5.1^{\mathrm{A}}\end{array}$

FGF-2-stimulated endothelial cells were treated with or without CA4P ( 10 and $50 \mathrm{nM}$ ) for 24 hours and analyzed for the presence of apoptotic cells by annexin $\mathrm{V}(\mathrm{AnnV}$ ) and propidium iodide ( $\mathrm{PI}$ ) staining using flow cytometry. Results of 3 experiments are expressed as the mean number of cells \pm SEM (AP $<0.001$ compared with CA4P-untreated cells; $n=3$ ).

the same site as colchicine does, but with even higher affinity $(20,21)$. Its prodrug, CA4P, has been shown to induce vascular shutdown in subcutaneous experimental tumors, causing tumor necrosis secondary to hemorrhage at concentrations well below the maximum tolerated dose, and to be more cytotoxic to rapidly proliferating rather than quiescent endothelial cells in vitro (22, 23). In vitro studies have shown that endothelial cells are particularly sensitive to the effects of CA4P compared with various other cell types. Proliferating human umbilical vein endothelial cells (HUVECs) round up when exposed to CA4P, and this is associated with condensation of the tubulin and reorganization of the actin cytoskeleton and with increased permeability of the endothelial cell monolayer to macromolecules $(24,25)$. Despite the remarkable effectiveness of CA4P in inducing vascular collapse in certain tumors, such as anaplastic thyroid cancers, dose-limiting toxicity and lack of understanding of its mechanism of action have delayed its clinical development. Specifically, it remains to be determined which antiangiogenic agents synergize with CA4P to enhance its therapeutic efficacy while diminishing its toxicity.

Indeed, the extent to which the tubulin-binding activity of CA4P accounts for its antivascular action remains unclear. In particular, the mechanism by which CA4P selectively targets and destabilizes tumor neovessels is not known. Here, we demonstrate that the antiangiogenic effect of CA4P is mediated through rapid disengagement and inhibition of the VE-cadherin/ $\beta$-catenin/Akt signaling pathway, leading to regression of tumor neovessels. As such, CA4P selectively targets pericyte-depleted tumor neovessels and opens new strategies to combine antivascular and antiangiogenic approaches for targeting tumor vasculature.

\section{Results}

CA4P inbibits endothelial cell proliferation. The mechanism whereby CA4P selectively targets tumor neovessels is not known. As endothelial cells recruited to tumor neovessels undergo rapid cell proliferation, we hypothesized that cytokine-stimulated endothelial cells may be more sensitive to the microtubule-destabilizing effects of CA4P. To test this hypothesis, we evaluated the kinetics of endothelial cell growth in the presence of angiogenic growth factors including FGF-2 and VEGF-A alone or in combination with various concentrations of CA4P $(0,1,5,10,20,30$, 40 , and $50 \mathrm{nM})$. We show that low concentrations of CA4P $(1 \mathrm{nM})$ were insufficient to block the proliferation of FGF-2- or VEGF-Astimulated endothelial cells. However, CA4P at nontoxic doses of $5 \mathrm{nM}$ and $10 \mathrm{nM}$ significantly decreased FGF-2- or VEGF-A-stim- ulated HUVEC proliferation $(P<0.05 ; n=4$; Figure $1 \mathrm{~A})$. Remarkably, CA4P at $1 \mathrm{nM}$ was more effective in inducing endothelial cell death when endothelial cells were stimulated with a combination of FGF-2 and VEGF-A $(P<0.01 ; n=4)$. The inhibitory effect of CA4P on endothelial cell proliferation was observed after 24 hours of treatment and was sustained until 48 hours. Doses of CA4P higher than $10 \mathrm{nM}$ had toxic effects, resulting in cell detachment promoting cell death. In contrast, CA4P had no effect on unstimulated endothelial cell proliferation at the concentration that significantly decreased the endothelial cell growth (i.e., $10 \mathrm{nM}$; Figure $1 \mathrm{~A})$. These data suggest that proliferating endothelial cells are more sensitive to the antivascular effects of CA4P.

To elucidate the mechanism by which CA4P exerts its antiproliferative effect on HUVECs, we investigated the effect of CA4P on inducing endothelial cell apoptosis. Subconfluent monolayers of HUVECs were incubated with FGF-2 in the presence and absence of CA4P $(0,10$, and $50 \mathrm{nM})$, and the number of apoptotic cells as quantified by annexin $\mathrm{V} /$ propidium iodide (annexin V/PI) staining was evaluated by flow cytometry. Surprisingly, a 24-hour incubation with CA4P did not induce endothelial cell death through apoptosis (Table 1). Similar results were obtained after 36 hours of treatment with CA4P, and short-time incubation with CA4P (12 hours) did not interfere with cell viability (data not shown). In addition, CA4P-induced endothelial cell death was not associated with activation of caspase- 3 or caspase-9, and the ratio of the level of the antiapoptotic protein $\mathrm{Bcl}-2$ to that of the proapoptotic protein Bax was not altered (data not shown). These data suggest that endothelial cell death mediated by CA4P treatment is not driven by classical apoptotic pathways.

HUVECs cocultured with SMCs are resistant to CA4P. Stability of fully assembled neovessels is supported by the recruitment of and periendothelial ensheathment with mural cells, including SMCs. As tumor neovessels are invested with disorganized sheaths of SMCs, we speculated that CA4P might also target SMCs. However, the survival of SMCs was not affected by CA4P even at high doses (such as $50 \mathrm{nM}$ ) that would induce significant endothelial cell death (Figure 1B). This finding raised the possibility that SMCs may confer endothelial cells with the capacity to resist cell death caused by CA4P. Remarkably, when cocultured with SMCs, HUVECs become resistant to CA4P (Figure 1C). These data suggest that SMCs confer endothelium with protection against CA4P-induced cell death, possibly through stabilization of endothelial cell-cell interaction.

CA4P blocks migration of neoangiogenic endothelial cells. Endothelial cell motility plays an essential role in supporting the survival and neoangiogenic phenotype of endothelial cells. CA4P as a prototypical anti-tubulin agent may not only block endothelial cell proliferation but also impair endothelial cell motility, leading to diminished signals promoting cell survival. To test this hypothesis, we challenged the capacity of FGF-2-stimulated endothelial cells to migrate into a mechanically denuded area. Indeed, 24 hours after wounding, FGF-2-treated endothelial cells could be seen to have migrated into the denuded area (Figure 1D). Remarkably, 10 nM CA4P completely blocked FGF-2-mediated endothelial cell migration into the denuded area as compared with the unstimulated- or FGF-2-stimulated HUVECs $(P<0.001$; $n=5$; Figure $1, \mathrm{D}$ and $\mathrm{E})$. These data suggest that CA4P, at a nontoxic dose, blocks endothelial cell migration and potentially leads to impaired tube formation.

CA4P impairs capillary tube formation but has no effect on SMC-stabilized tubes. Capillary tube formation is a multistep process involv- 


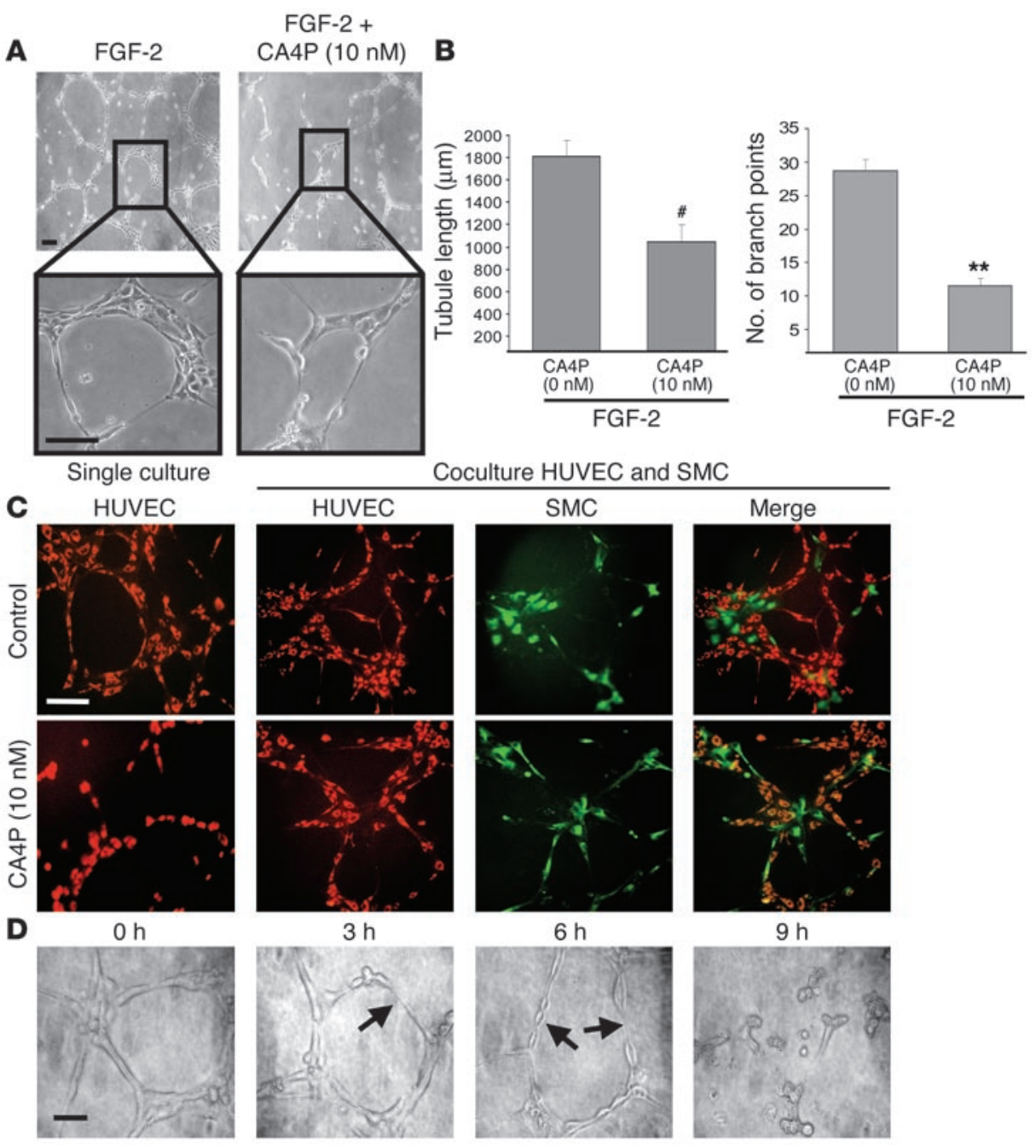

Figure 2

CA4P impairs capillary tube formation, but has no effect on SMC-stabilized tubes, and destabilizes a preestablished vascular network. (A) CA4P inhibits capillary tube formation. HUVECs $\left(3 \times 10^{4}\right)$ were seeded on Matrigel matrix and incubated in the presence of FGF-2 and with $10 \mathrm{nM} \mathrm{CA4P.} \mathrm{The} \mathrm{effect}$ of CA4P on capillary tube formation was observed after a 12-hour incubation under an inverted light microscope. Magnification: $\times 10$ (top panels), $\times 40$ (bottom panels). Scale bar, $10 \mu \mathrm{m}$ (top panels), $15 \mu \mathrm{m}$ (bottom panels). (B) Quantification of the CA4P-mediated capillary tube formation inhibition. The quantification was done by measuring the tubule length and counting the number of branch points in 4 different random pictures. Results are expressed as the mean of the tubule length and the mean of the number of branch points \pm SEM ${ }^{* *} P<0.01,{ }^{*} P<0.001$ compared with CA4P-untreated cells; $\left.n=4\right)$. (C) SMCs protect capillary tube formation against CA4P. HUVECs $\left(3 \times 10^{4}\right)$ stained with red fluorescent cell linker and SMCs $\left(1 \times 10^{4}\right)$ stained with green fluorescent cell linker were seeded together on Matrigel matrix and incubated in presence of FGF-2 and with $10 \mathrm{nM} \mathrm{CA4P.} \mathrm{The} \mathrm{effect} \mathrm{of} \mathrm{CA4P}$ on capillary tube formation was observed after a 12-hour incubation under an inverted fluorescent microscope. Magnification: $\times 20$. Scale bar, $50 \mu \mathrm{m}$. (D) CA4P destabilizes a preestablished vascular network. HUVECs $\left(3 \times 10^{4}\right)$ were seeded on Matrigel matrix and incubated in presence of FGF-2. Once the capillary network formed (after 12 hours), CA4P was added, and the effect of $10 \mathrm{nM} \mathrm{CA} 4 \mathrm{P}$ on the disruption of the capillary network was monitored in a time-course manner every 3 hours. There was significant disruption of endothelial cell morphology (arrows). Magnification: $\times 40$. Scale bar, $10 \mu \mathrm{m}$.

ly shown in the proliferation assay. FGF-2-stimulated endothelial cells induced the formation of a polygonal capillary network, which was destabilized in presence of $10 \mathrm{nM} \mathrm{CA4P} \mathrm{(Fig-}$ ure 2A). CA4P significantly decreased the tubule length and the formation of branch points as compared with FGF-2-stimulated endothelial cells (Figure 2B). Since SMCs have been shown to protect HUVECs against CA4P-induced cell death, the capacity of endothelial cells to form capillary tubes was evaluated in the presence of SMCs and CA4P. Remarkably, HUVECs cocultured with SMCs in the presence or absence of $10 \mathrm{nM}$ CA4P were able to assemble into capillary tubes, in which endothelial cells were stabilized with SMCs (Figure 2C). In contrast, pure cultures of endothelial cells treated with CA4P failed to assemble into capillary tubes. These data demonstrate that mural protection of endothelial cells might confer protection of vascular tubes against CA4P-induced vessel destabilization.

We then evaluated the effect of CA4P on preestablished capillary networks grown in the presence of FGF-2. As shown in Figure 2D, incubation of endothelial cells with FGF-2 supported the generation of a cellular capillary network. However, after a 3-hour treatment with $10 \mathrm{nM}$ CA4P, the preestablished vessels were disrupted, as evidenced by the formation of round and disorganized sheaths of endothelial cells (Figure 2D, arrow). By 9 hours, endothelial cells were disengaged, dropping out of the typical capillary lumen. These data indicated that CA4P might impair the shape of endothelial cells through destabilization of the cytoskeleton, thereby depriving them of junctional survival signals conveyed by the endothelial-specific adherens junction, such as VE-cadherin.

CA4P induces endothelial cell retraction and actin depolymerization. To determine the mechanism by which CA4P may alter the shape of endothelial cells, the effect of CA4P on FGF-2stimulated HUVECs was studied. ing cell adhesion and migration and remodeling of proliferating endothelial cells. As CA4P interferes with endothelial cell proliferation and motility, we hypothesized that CA4P may also disrupt tube formation. For this purpose, we used a dose of $10 \mathrm{nM}$ CA4P, which is the optimal physiologically relevant concentration to block angiogenesis under in vitro conditions, as previous-
In the first 18 hours following exposure to $5 \mathrm{nM}$ and $10 \mathrm{nM}$ of CA4P, endothelial cells remained flat (Figure 3A). However, after 18 hours of treatment with CA4P, the endothelial cells retracted and rounded up and by 24 hours detached from the culture plate and underwent cell death (Figure 3A). The effect of CA4P was then assessed on actin cytoskeleton. After 18 hours of incubation 
A

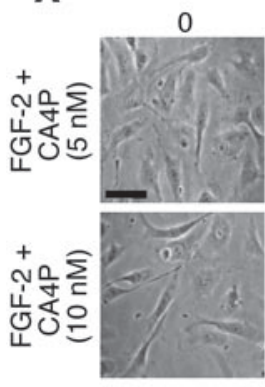

B

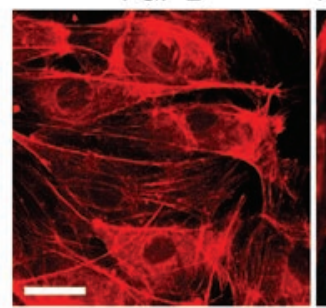

6

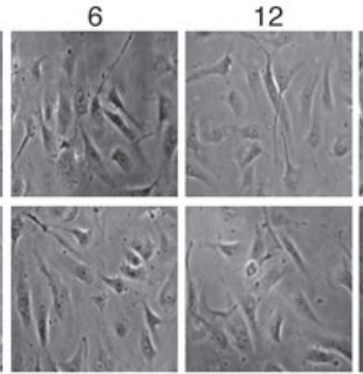

FGF-2 + CA4P (2.5 nM)
Time (h)
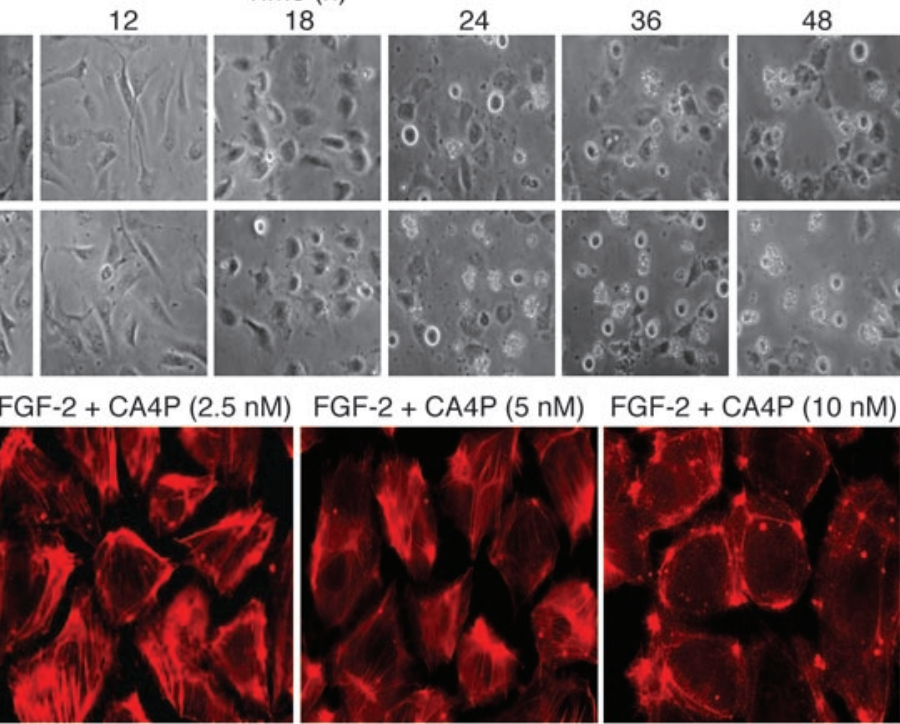

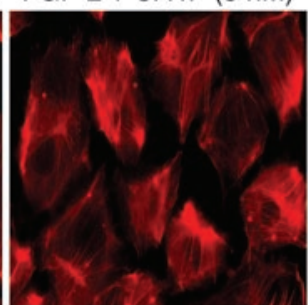

$F G F-2+C A 4 P(10 n M)$

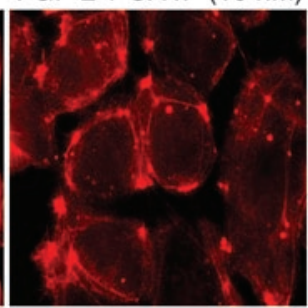

\section{Figure 3}

CA4P induces retraction of endothelial cells and disrupts actin cytoskeleton. (A) CA4P induces retraction of endothelial cells. The effect of CA4P (5 nM and $10 \mathrm{nM}$ ) on the cell shape of FGF-2-stimulated HUVECs was monitored during 48 hours by microscopy. Notice the retraction of HUVECs after 18 hours of treatment with CA4P. Magnification: $\times 20$. Scale bar, $10 \mu \mathrm{m}$. (B) The actin cytoskeleton was stained with TRITC-labeled phalloidin and analyzed by fluorescent microscopy on FGF-2-stimulated HUVECs after an 18-hour incubation with CA4P. Addition of CA4P resulted in reorganization of actin stress fibers. Magnification: $\times 40$. Scale bar, $5 \mu \mathrm{m}$. with $2.5 \mathrm{nM}$ CA4P, the endothelial cells rapidly lost their cell-cell contact without affecting the organization of the actin fibers (Figure 3B). Remarkably, $5 \mathrm{nM}$ CA4P interfered with the alignment of actin stress fibers, and the organization of the actin cytoskeleton was completely disrupted at higher concentration of CA4P $(10 \mathrm{nM})$, resulting in the disappearance of actin fibers and their relocation and reorganization in the intracellular focal adhesion regions. As VE-cadherin anchors and interacts with actin cytoskeleton via $\beta$-catenin, it is conceivable that CA4P interference with the organization of endothelial stress fibers may contribute to disengagement of homophilic VE-cadherin/ $\beta$-catenin-mediated cell-cell interactions.

The antivascular effect of $C A 4 P$ is mediated through rapid inbibition and disengagement of VE-cadherin/ $\beta$-catenin function. Since CA4P disrupts endothelial junctional integrity, we tested the hypothesis that CA4P could interfere with VE-cadherin homophilic interactions. To this end, HUVECs were stimulated with FGF-2 in the presence of $10 \mathrm{nM}$ CA4P. During the first hour of treatment with CA4P, $\mathrm{VE}$-cadherin was focally localized to the interjunctional region of endothelial cells, assuming an organized linear pattern (Figure 4A). Remarkably, after 3-6 hours of exposure to CA4P, the VE-cadherin staining at cell-cell contacts redistributed into a disorganized zigzag pattern $(P<0.001 ; n=10$; Figure 4, A [arrowheads] and $\mathrm{B})$, which has been previously shown to be the hallmark of VE-cadherin disengagement (26). However, 12 hours after CA4P treatment, the redistributed VE-cadherin at the cell-cell contacts appeared to be associated with the partitioned and separated gaps between the endothelial cells (Figure 4A, asterisks), which were more visible after 18 hours of treatment, suggesting a loss of intercellular contacts. The rapid initial VE-cadherin redistribution that occurred before cell retraction was concomitant with the redistribution of $\beta$-catenin, a signaling partner of VE-cadherin. In fact, staining for $\beta$-catenin showed similar disrupted zigzag pattern at the same time points, i.e., after 3 and 6 hours of CA4P treatment $(P<0.001 ; n=10$; Figure 4, A [arrowheads] and B), leading to the formation of separated gaps (Figure 4A, asterisks).

To assess whether CA4P may also disrupt the distribution of other junctional proteins, the localization of the junctional pro- teins PECAM-1, zona occludens 1 (ZO-1), occludin, and connexin-43 was investigated on endothelial cells upon treatment with $10 \mathrm{nM}$ CA4P. Although there was no apparent disruption of these junctional molecules (Supplemental Figure 1; supplemental material available online with this article; doi:10.1172/JCI24586DS1), it remains to be determined whether CA4P has any major effect on functional engagement of these junctional molecules with the intracellular signaling components. Notwithstanding, since among the known adherens molecules VE-cadherin is the key endothelialspecific junctional protein that is essential for the integrity and survival of endothelial cells, we focused our attention on the mechanism by which CA4P may exert its potent antivascular effect through destabilization of the VE-cadherin/ $\beta$-catenin complex leading to cell death.

To formally prove that the inhibitory effect of CA4P on endothelial cell homophilic interaction is mediated through VEcadherin disengagement, we assessed the effect of CA4P alone or in combination with a neutralizing $\mathrm{mAb}$ against human $\mathrm{VE}$-cadherin (clone BV9) on adenoviral E4 ${ }^{+}\left(\mathrm{AdNullE}^{+}\right)$vector-infected HUVECs. We have shown that introduction of adenovirus E4 gene into the endothelial cells stabilizes VE-cadherin/ $\beta$-catenin homophilic interactions, thereby augmenting the survival of endothelial cells through an Akt-dependent pathway (27). Remarkably, AdNullE4+-, but not control AdNullE4--, infected HUVECs, were resistant to CA4P, and cells became sensitive to CA4P treatment only when high concentrations of CA4P $(50 \mathrm{nM})$ were used $(P<0.001 ; n=4$; Figure 4C). Neutralizing mAb against VE-cadherin also impaired endothelial cell viability at a high concentration $(10 \mu \mathrm{g} / \mathrm{ml})(P<0.05 ; n=4)$. Cotreatment of AdNullE4 ${ }^{+}-$ infected HUVECs with CA4P and $\mathrm{mAb}$ against VE-cadherin greatly increased the sensitivity of AdNullE4+-transduced HUVECs, since introduction of $10 \mathrm{nM}$ of CA4P concomitant with $2.5 \mu \mathrm{g} / \mathrm{ml}$ of $\mathrm{mAb}$ against $\mathrm{VE}$-cadherin significantly decreased endothelial cell viability $(P<0.01 ; n=4)$. These data support the notion that disruption of VE-cadherin by CA4P plays a critical role in promoting endothelial cell death.

To determine whether the CA4P-induced loss of cell-cell interaction and disruption of VE-cadherin/ $\beta$-catenin complex 
A
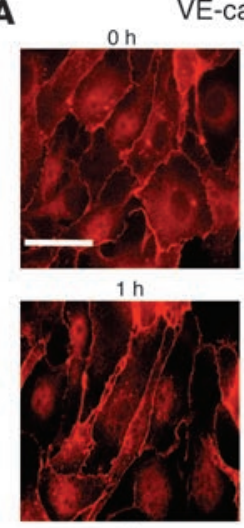

$3 \mathrm{~h}$
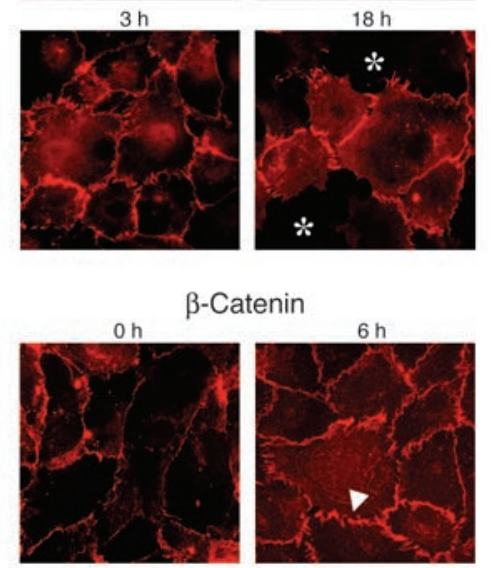

$\beta$-Catenin
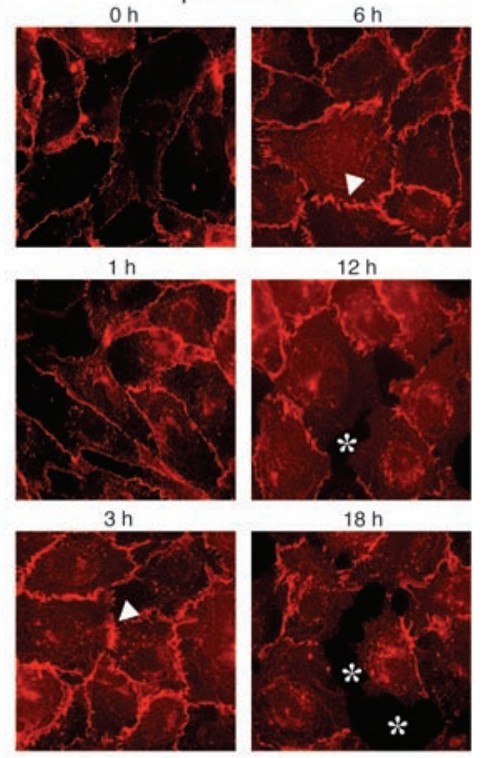

B

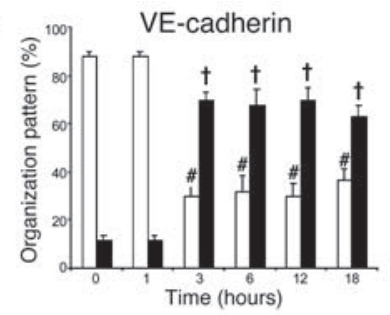

C
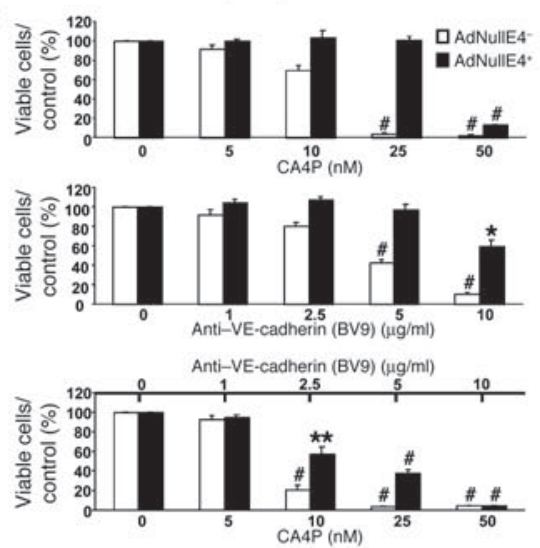

D

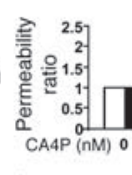

$3 \mathrm{~h}$

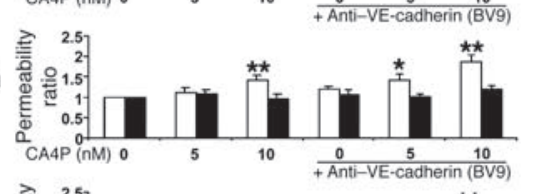

$6 \mathrm{~h}$

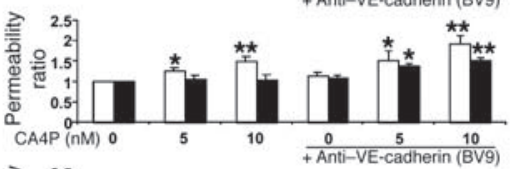

$12 \mathrm{~h}$

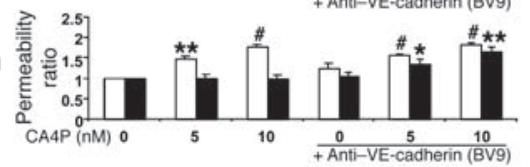

$18 \mathrm{~h}$

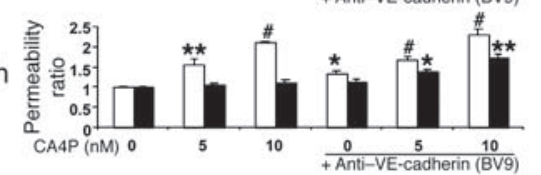

$\beta$-Catenin

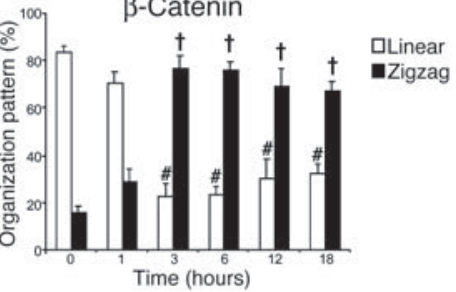

E

IP: VE-cadherin

IB: phosphotyrosine and VE-cadherin

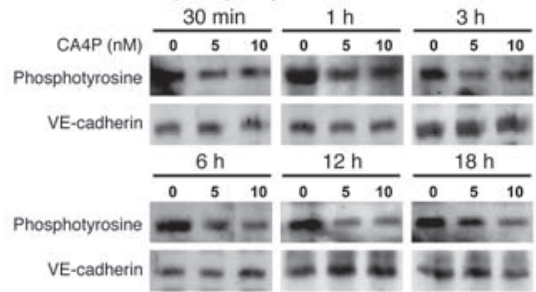

IP: $\beta$-catenin

IB: phosphotyrosine and $\beta$-catenin

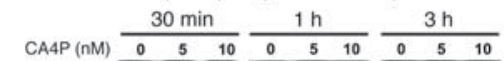
Phosphotyrosine P F

$\beta$-Catenin $0=-\infty-\infty$

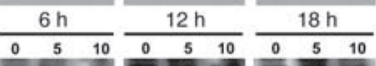

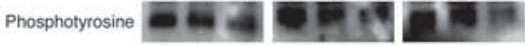
$\beta$-Catenin $-\infty 0-\infty-\infty$

$\mathbf{F}$

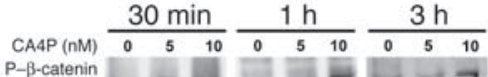
P- $\beta$-catenin
Ser45 $\underset{\mathrm{P} 33 / 37 / \mathrm{Thr} 41}{\mathrm{P}-\text {-catenin }}-\mathrm{t}-1-\cdots \rightarrow$

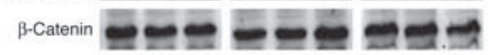
$\frac{6 h}{0} \frac{12 h}{0} \frac{18 h}{0}$ P- $\beta$-catenin
Ser45 P-B-catenin
S33/37/Thr41

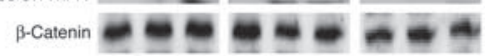

G

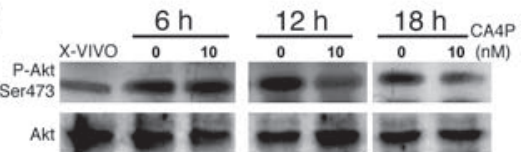

Figure 4

CA4P blocks VE-cadherin/ $\beta$-catenin/Akt signaling pathway. (A) CA4P disengages VE-cadherin and $\beta$-catenin. The localization of VE-cadherin and $\beta$-catenin in FGF-2-stimulated HUVECs was monitored during 18 hours by microscopy upon CA4P treatment (10 nM). Notice the redistribution of VE-cadherin and $\beta$-catenin assuming a zigzag pattern at cell-cell contacts (arrowheads) before the apparition of gaps between the cells (asterisks). Magnification: $\times 40$. Scale bar, $10 \mu \mathrm{m}$. (B) Quantification of the organization pattern of VE-cadherin and $\beta$-catenin. The organization pattern, i.e., linear or zigzag, was evaluated by microscopic counting of 10 fields at $\times 10$ magnification and presented as the percentage of linear or zigzag pattern per microscopic field \pm SEM ( ${ }^{\#} P<0.001,{ }^{\dagger} P<0.001$ compared with linear and zigzag organization pattern observed at time 0 , respectively; $\left.n=10\right)$. (C) CA4P synergizes with human mAb against VE-cadherin to decrease cell viability. AdNullE4-- or AdNullE4+-infected HUVECs were incubated with either CA4P or human mAb against VE-cadherin (BV9) or in combination, and the number of viable cells was determined after a 48-hour incubation. Results of 4 experiments in duplicate are expressed as the mean number of viable cells $\pm \mathrm{SEM}\left({ }^{\star} P<0.05,{ }^{\star \star} P<0.01\right.$, $\# P<0.001 \mathrm{compared}$ with untreated cells; $n=4$ ). (D) CA4P synergizes with neutralizing mAb against VE-cadherin to increase endothelial cell permeability. Modification of endothelial cell permeability of AdNullE4-- or AdNullE4+-infected HUVEC monolayers was assessed as described in Methods at designated time points. Results of 3 experiments in triplicate are expressed as the permeability ratio (experimental/control) \pm SEM $\left({ }^{\star} P<0.05\right.$, ${ }^{* \star} P<0.01$, ${ }^{\#} P<0.001$ compared with control; $n=3$ ). (E) CA4P inhibits tyrosine phosphorylation of VE-cadherin and $\beta$-catenin. Levels of tyrosine phosphorylation of immunoprecipitated VE-cadherin and $\beta$-catenin were determined by Western blotting. (F) CA4P induces serine/threonine phosphorylation of $\beta$-catenin. Phosphorylation levels (Ser45 and Ser33/37/Thr41) of $\beta$-catenin were determined by Western blotting. (G) CA4P decreases Akt phosphorylation. Phosphorylation level (Ser 473) of Akt was determined by Western blotting. The condition where the serum-free medium (X-VIVO) was used corresponds to basal protein expression in the absence of stimulation. 

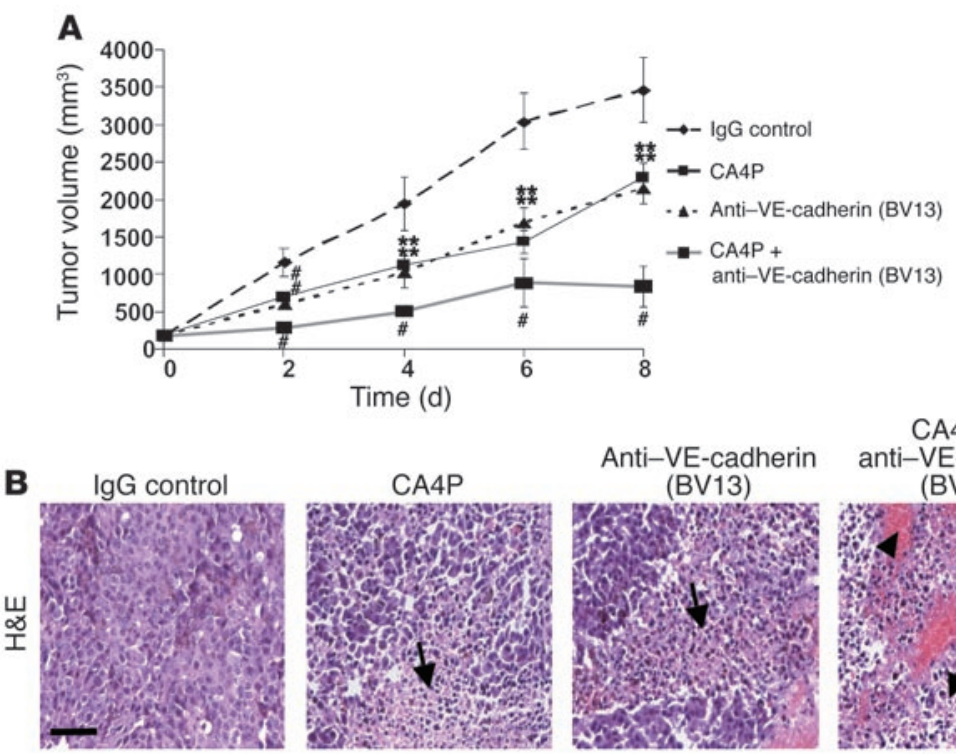

C

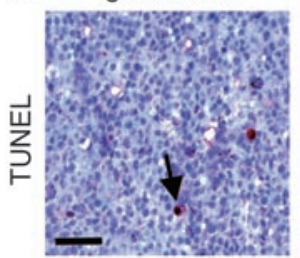

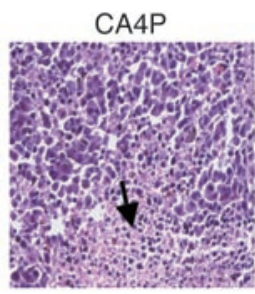

Anti-VE-cadherin (BV13)

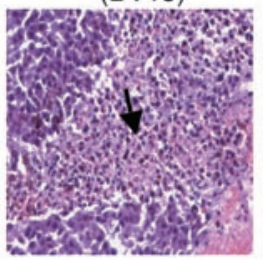

Anti-VE-cadherin (BV13)
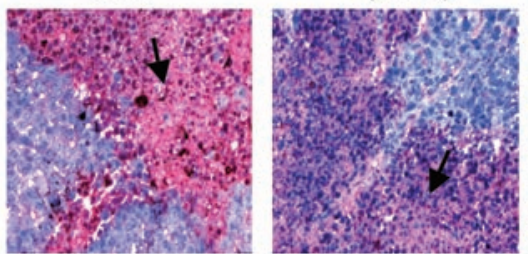

$\mathrm{CA} 4 \mathrm{P}+$ anti-VE-cadherin (BV13)

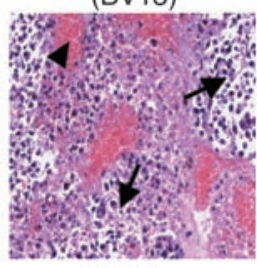

$\mathrm{CA} 4 \mathrm{P}+$ anti-VE-cadherin (BV13)

\section{Figure 5}

Treatment with CA4P and neutralizing $\mathrm{mAb}$ against VE-cadherin (BV13) promotes B16 melanoma tumor necrosis. (A) Growth curves of IgG control-, CA4P-, anti-VE-cadherin- (BV13), or CA4P plus anti-VEcadherin- (BV13) treated tumors. B16 melanoma cells were injected subcutaneously into the dorsa of C57BL/6 mice. Treatment was initiated after 10 days and mice injected every 2 days. Mice in the CA4P group received an i.v. injection of CA4P at $5 \mathrm{mg} / \mathrm{kg}$; the anti-VE-cadherin group received an i.p. injection of $10 \mu \mathrm{g}$ of $\mathrm{mAb}$ against VE-cadherin (BV13); the combined group received CA4P at $5 \mathrm{mg} / \mathrm{kg}$ plus $10 \mu \mathrm{g}$ of mAb against VE-cadherin (BV13); and the control group received $10 \mu \mathrm{g}$ of IgG antibody. Tumor size was measured until day 8 after the first injection, and tumor volumes were measured. The data represent the mean value of the tumor volume \pm SEM ( ${ }^{\star \star} P<0.01,{ }^{\#} P<0.001$ compared with IgG control group; $n=9$ ). (B) Histopathological analysis of CA4Pand anti-VE-cadherin- (BV13) treated B16 melanoma tumors. Animals were sacrificed at day 8 after first injection. Shown here are H\&E-stained paraffin sections demonstrating areas of necrosis (arrows) with CA4P and mAb against VE-cadherin (BV13) treatment. Arrowhead shows area of hemorrhage. Magnification: $\times 20$. Scale bar, $80 \mu \mathrm{m}$. (C) Induction of tumor necrosis. Cell death within paraffin tumor sections was detected by TUNEL. Red staining represents positive signals within the tumors (blue cells are the negative, living cells). Arrows show areas of necrosis. Magnification: $\times 20$. Scale bar, $80 \mu \mathrm{m}$. would result in reduced monolayer integrity, we assessed the effect of CA4P on the permeability of AdNullE4+- and control AdNullE4--infected HUVEC monolayers. Remarkably, as compared with AdNullE4--infected HUVECs, monolayers of AdNullE4+-infected HUVECs were resistant to CA4P and did not manifest a significant increase in permeability even after 18 hours of treatment with CA4P (Figure 4D). However, control AdNullE4- endothelial cell monolayers became permeable within 3 hours of treatment with CA4P ( $<<0.05 ; n=3$; Figure 4D). Treatment with neutralizing $\mathrm{mAb}$ against $\mathrm{VE}$-cadherin alone also impaired endothelial cell permeability, but only after 18 hours of incubation $(P<0.05 ; n=3$; Figure $4 \mathrm{D})$. In line with the previous finding that CA4P synergizes with $\mathrm{mAb}$ against VEcadherin, the endothelial cell monolayer permeability was significantly increased by treatment with a combination of CA4P and $\mathrm{mAb}$ against $\mathrm{VE}$-cadherin $(P<0.05 ; n=3$; Figure $4 \mathrm{D})$. Taken together, these results set forth the notion that CA4P, in part through dysregulation of VE-cadherin homotypic interaction, impairs the endothelial cell barrier function, since AdNullE4+infected HUVEC monolayers where VE-cadherin/ $\beta$-catenin homophilic interactions are tightly stabilized were not permeable upon CA4P treatment, while control AdNullE4- endothelial cell monolayers were highly sensitive to CA4P treatment.

The mechanism by which CA4P modulates VE-cadherin/ $\beta$-catenin function was further dissected at different time points ( 30 minutes and $1,3,6,12$, and 18 hours) in the presence or absence of $5 \mathrm{nM}$ and $10 \mathrm{nM} \mathrm{CA4P.} \mathrm{The} \mathrm{levels} \mathrm{of} \mathrm{tyrosine} \mathrm{phosphorylation} \mathrm{of} \mathrm{VE-cad-}$ herin and $\beta$-catenin were assessed by immunoprecipitation of VEcadherin and $\beta$-catenin, respectively, followed by blotting for phos- photyrosine. Remarkably, CA4P rapidly decreased VE-cadherin and $\beta$-catenin tyrosine phosphorylation after 1 hour of treatment, and this reduction was maintained until 18 hours (Figure 4E). We further questioned whether CA4P could interfere with VE-cadherin function, possibly through modulating $\beta$-catenin phosphorylation status. The endothelial cell lysates were examined using anti-phospho- $\beta$-catenin Ser45 antibody and anti-phospho- $\beta$-catenin Ser33/37/Thr41 antibody, which recognizes $\beta$-catenin when it is phosphorylated at 1 or more of 3 specific sites, namely Ser33, Ser37, or Thr41. Remarkably, CA4P induced the phosphorylation of $\beta$-catenin on both Ser 45 and Ser33, Ser37, or Thr41 after 1 hour of treatment (Figure 4F). Moreover, the increase in phospho- $\beta$-catenin Ser33/37/Thr41 was confirmed by immunostaining of CA4P-treated endothelial cells (Supplemental Figure 2). These findings suggest that CA4P may interfere with $\beta$-catenin function, since phosphorylation of serine/threonine residues in the $\mathrm{N}$-terminal region of $\beta$-catenin targets the protein for ubiquitination and subsequent proteasomal degradation $(28,29)$. There was no change in the total amount of the VE-cadherin and $\beta$-catenin, suggesting that the observed phosphorylation patterns are independent of the changes in the overall protein expression.

The decline in the tyrosine phosphorylation of VE-cadherin/ $\beta$-catenin complex and increased serine/threonine phosphorylation of $\beta$-catenin were detected much earlier than the induction of cell retraction, which was observed after 18 hours of treatment with CA4P (Figure 3A). These data set forth the notion that rapid CA4Pmediated inhibition of VE-cadherin/ $\beta$-catenin disengagement and function contribute to the selective destabilization of endothelial cell-cell junctions, which is the early event in inducing the loss of anchorage of endothelial cells, setting the stage for cell death. 


\section{Figure 6}

CA4P synergizes with $\mathrm{mAb}$ against VE-cadherin (BV13) to block tumor neoangiogenesis. C57BL/6 mice bearing B16 melanoma cells were treated with IgG control, CA4P, anti-VEcadherin (BV13), or CA4P plus anti-VE-cadherin (BV13) as described in Methods. After an 8-day treatment, tumors were removed and subjected to immunohistochemical analysis. (A) Vessel density determination in B16 melanoma tumors. The importance of intratumoral vascularization was assessed by PECAM-1 immunostaining (red fluorescence). Nuclei were detected by DAPI staining (blue). Note that numerous vessels are seen in the control group, whereas groups of mice treated with CA4P, anti-VE-cadherin (BV13), or combination have markedly fewer neovessels. Representative tumor sections of each group are shown. Magnification: $\times 20$. Scale bar, $100 \mu \mathrm{m}$. (B) Quantification of the microvessel density in B16 melanoma tumor sections. The microvessel density is presented as mean number of microvessels per microscopic field \pm SEM $\left({ }^{*} P<0.05,{ }^{\#} P<0.001\right.$ compared with the IgG control group; $n=5$ ). (C) CA4P impairs SMCmediated stabilization of neovessels. The presence of endothelial cells and SMCs was assessed by fluorescence microscopy using PECAM-1 (red) and $\alpha$-SMA (green) staining, respectively. Note that vessels in the CA4P and CA4P plus anti-VE-cadherin (BV13) groups are not positive for $\alpha$-SMA, whereas in the absence of CA4P, vessels are surrounded by SMCs. Representative photographs of tumor sections are shown. Magnification: $\times 100$. Scale bar, $10 \mu \mathrm{m}$. (D) Quantification of SMC-ensheathed vessels in B16 melanoma tumor sections. The number of SMC-ensheathed vessels is presented as mean number of SMC-ensheathed vessels per microscopic field \pm SEM $\left({ }^{\#} P<0.001\right.$ compared with the IgG control group; $n=5$ ).
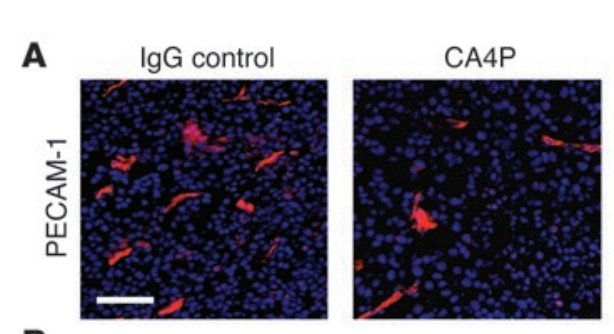

Anti-VE-cadherin (BV13)
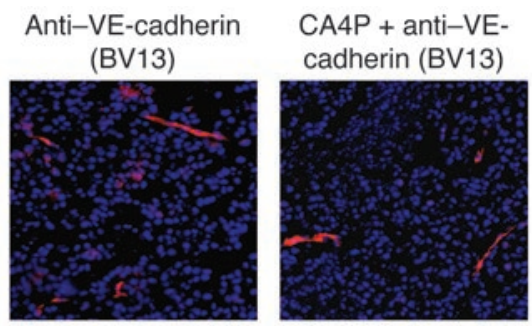

B

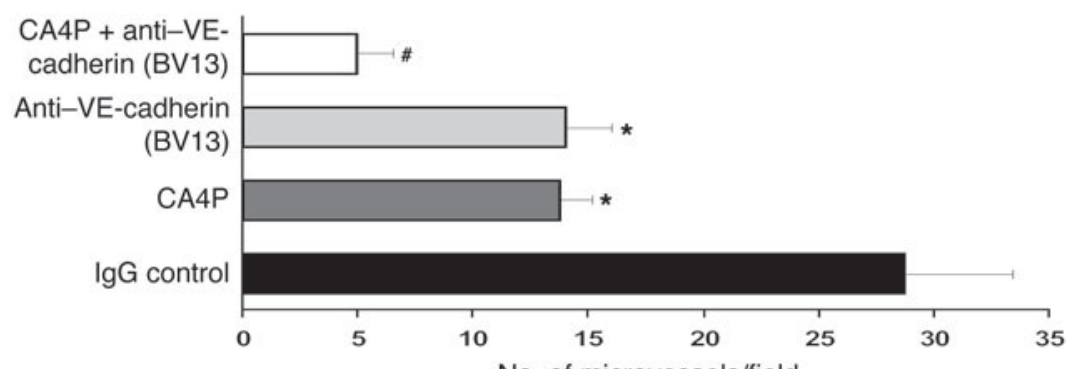

No. of microvessels/field Anti-VE-cadherin $\quad$ CA4P + anti-VE-

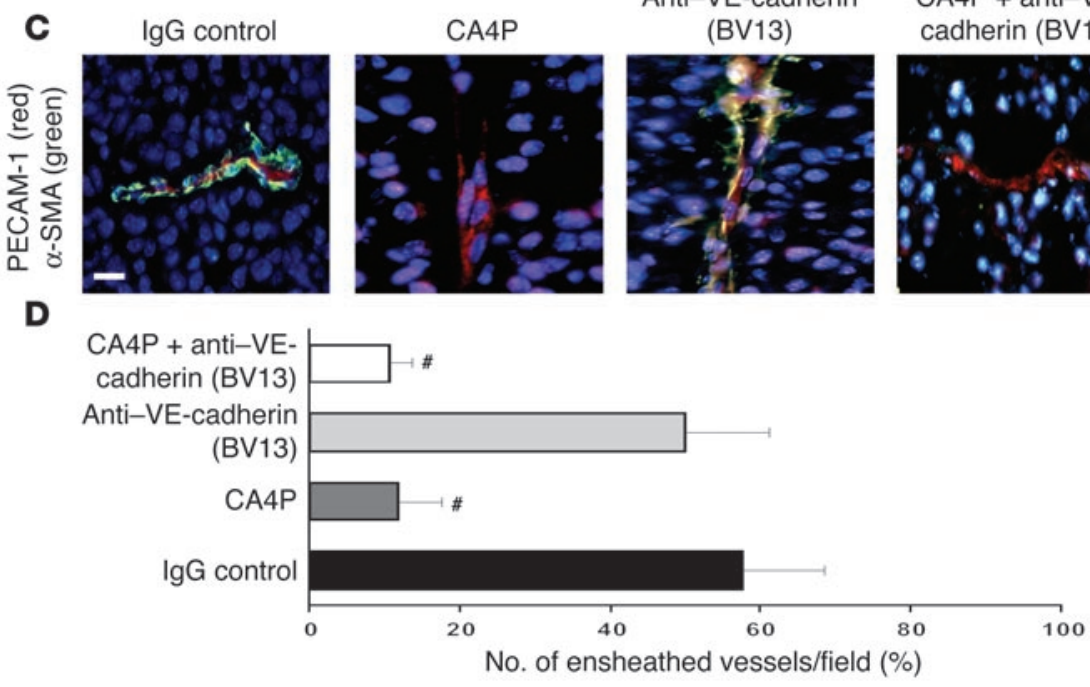

CA4P decreases the phosphorylation of Akt. As the VE-cadherin/ $\beta$-catenin complex is upstream of the PI3K/Akt signaling pathway, we further examined whether CA4P inhibits FGF-2-mediated Akt phosphorylation. The incubation of FGF-2-stimulated endothelial cells with CA4P did not affect the phosphorylation of Akt (Ser473) during the first 6 hours of treatment (Figure 4G). However, treatment with $10 \mathrm{nM}$ CA4P decreased the phospho-Akt level after a 12-hour incubation, and the level of phospho-Akt was comparable to that observed for unstimulated cells incubated under serumfree conditions. CA4P-mediated inhibition of Akt phosphorylation was maintained after an 18-hour incubation with CA4P. This reduction was clearly related to a decrease in phosphorylation, since the total amount of Akt was not altered by CA4P treatment (Figure 4G). Taken together, these data suggest that the rapid antivascular (12 hours after exposure to CA4P) effect of CA4P on growth factor-stimulated endothelial cells is primarily mediated through destabilization of VE-cadherin leading to inhibition of $\beta$-catenin and PI3K/Akt cell signaling.

$C A 4 P$ synergizes with neutralizing $m A b$ against VE-cadherin to block tumor neoangiogenesis. Angiogenic factors and physical forces imparted by the periendothelial cells activate signaling molecules that directly stabilize VE-cadherin homotypic interaction, thereby counteracting the destabilizing effect of CA4P on engaged VE-cadherin. Therefore, forced physical disengagement of VE-cadherin by neutralizing antibodies may facilitate the cytoskeletal destabilizing effect of CA4P. To this end, the potential synergistic effect of CA4P and forced VE-cadherin disruption on blocking tumor neoangiogenesis was tested by introducing CA4P and/or neutralizing mAb against VE-cadherin into mice with preestablished B16 melanoma tumors. CA4P injections were performed every 2 days, and tumor growth was monitored for 8 days after the beginning of the treatment. As shown in Figure 5A, CA4P treatment induced a significant delay in tumor growth as compared with the control group 3 days after treatment $\left(902.26 \pm 47.50 \mathrm{~mm}^{3}\right.$ versus $1,542.26 \pm 304.06 \mathrm{~mm}^{3}$; $P<0.001 ; n=9)$. Intravenous injection of neutralizing $\mathrm{mAb}$ against murine VE-cadherin (BV13), at low doses to avoid end-organ toxicity (6), reduced tumor growth as compared with the control group $\left(766.16 \pm 138.16 \mathrm{~mm}^{3}\right.$ versus $1,542.26 \pm 304.06 \mathrm{~mm}^{3} ; P<0.001$; $n=9)$. The degree of inhibition of tumor growth was comparable to that achieved with CA4P treatment. The stabilization of tumor growth was maintained until the end of the treatment period $(P<0.01 ; n=9)$. However, the maximum tumor growth inhibition 


\section{Figure 7}

CA4P synergizes with $\mathrm{mAb}$ against VE-cadherin (E4G10) to block tumor neoangiogenesis. C57BL/6 mice bearing B16 melanoma cells were treated with IgG control, CA4P, anti-VEcadherin (E4G10), or CA4P plus anti-VE-cadherin (E4G10) as described in Methods. After an 8-day treatment, tumors were removed and subjected to immunohistochemical analysis. (A) Growth curves of IgG control-, CA4P-, antiVE-cadherin- (E4G10), or CA4P plus anti-VEcadherin- (E4G10) treated tumors. Tumor size was measured until day 8 after first injection. The data represent the mean value of the tumor volume \pm SEM $\left({ }^{* *} P<0.01\right.$, ${ }^{*} P<0.001$ compared with IgG control group; $n=9$ ). (B) Histopathological analysis of CA4P- and anti-VE-cadherin(E4G10) treated B16 melanoma tumors. Shown here are $\mathrm{H} \& \mathrm{E}$-stained sections demonstrating areas of necrosis (arrows) with CA4P and $\mathrm{mAb}$ against VE-cadherin (E4G10) treatment. Arrowhead shows area of hemorrhage. Magnification: $\times 20$. Scale bar, $80 \mu \mathrm{m}$. (C) Vessel density determination in B16 melanoma tumors. The importance of intratumoral vascularization was assessed by PECAM-1 immunostaining (red fluorescence). Nuclei were detected by DAPI staining (blue). Note that numerous vessels are seen in the control group, whereas groups of mice treated with CA4P, anti-VE-cadherin (E4G10), or combination have markedly fewer neovessels. Representative tumor sections of each group are shown. Magnification: $\times 20$. Scale bar, $100 \mu \mathrm{m}$. (D) Quantification of the microvessel density in B16 melanoma tumor sections. The microvessel density is presented as mean number of microvessels per microscopic field \pm SEM $\left({ }^{*} P<0.05,{ }^{\#} P<0.001\right.$ compared with the IgG control group; $n=5$ ).
A
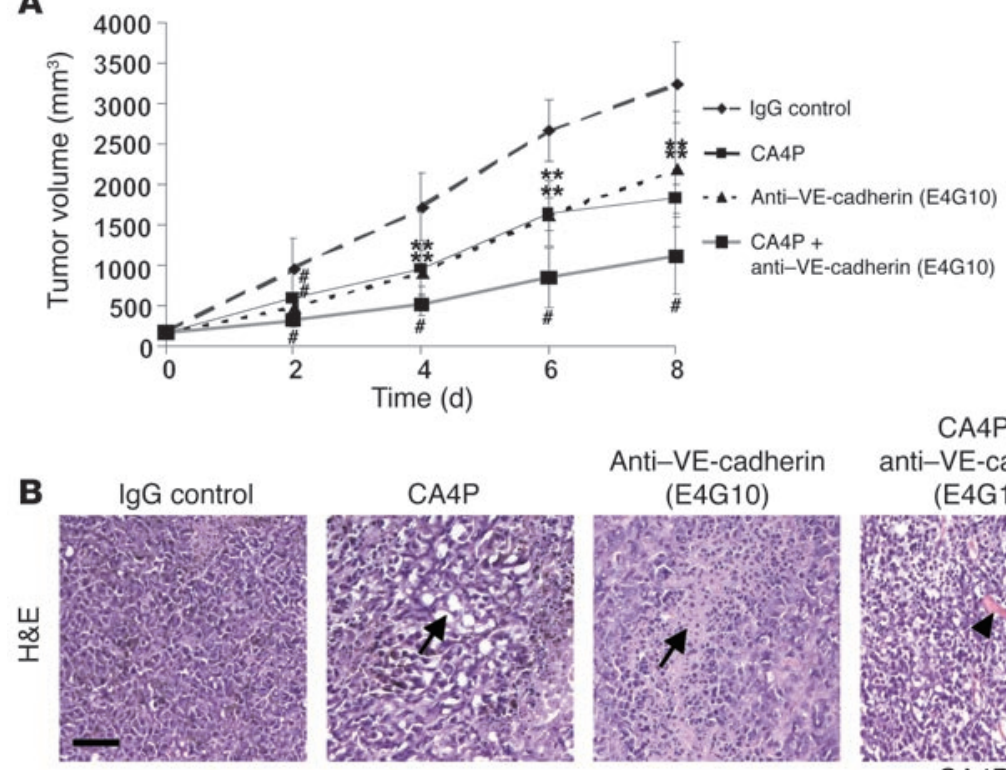

Anti-VE-cadherin

$\mathrm{CA} 4 \mathrm{P}+$

CA4P

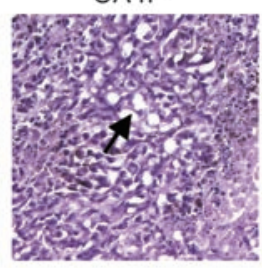

(E4G10)

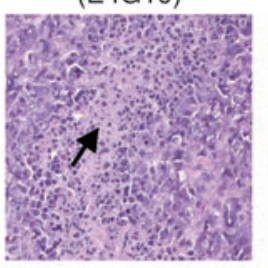

anti-VE-cadherin

(E4G10)

C

IgG control

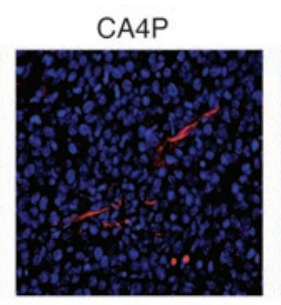

Anti-VE-cadherin (E4G10)

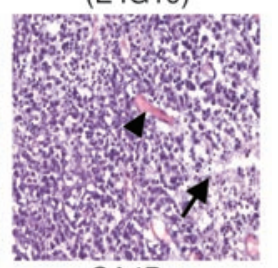

CA4P +

anti-VE-cadherin
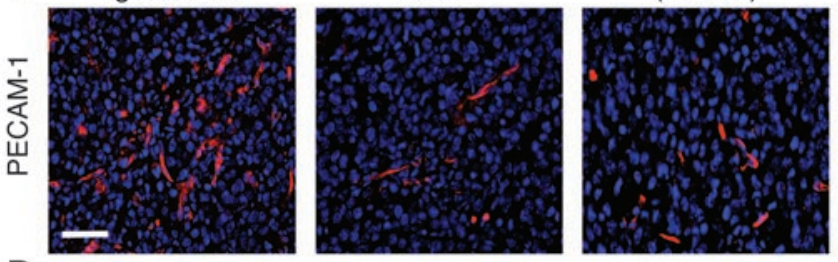

(E4G10)

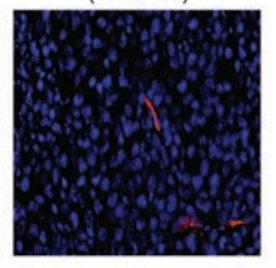

D

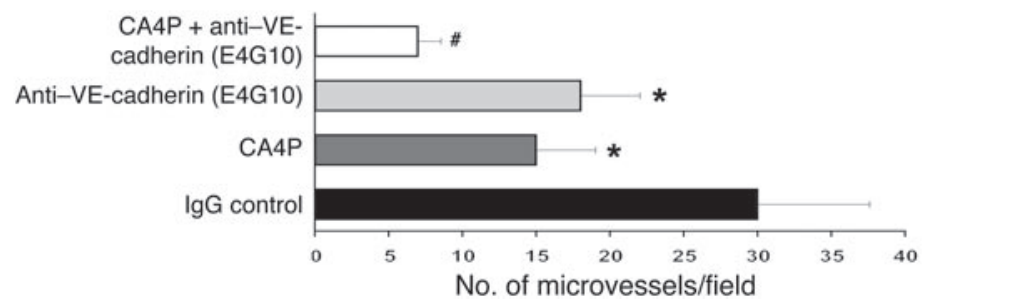

was achieved by treating the inoculated mice with a combination of CA4P and $\mathrm{mAb}$ against VE-cadherin (BV13). Indeed, at the end of the treatment period (i.e., at day 8), tumor growth was reduced by $75 \%$ in mice treated with CA4P and $\mathrm{mAb}$ against VE-cadherin (BV13) as compared with the control group $\left(839.10 \pm 271.16 \mathrm{~mm}^{3}\right.$ versus $\left.3,462.37 \pm 437.51 \mathrm{~mm}^{3} ; P<0.001 ; n=9\right)$.

Histological examination of tumor sections showed large areas of viable melanoma cells without significant necrosis or fibrosis formation in the untreated control mice (Figure 5B). In contrast, multiple necrotic areas within the tumors were detected in groups of mice that were treated with either CA4P or mAb against VEcadherin (BV13) (Figure 5B, arrows). There was significantly more necrosis in the mice that were treated with both $\mathrm{CA} 4 \mathrm{P}$ and $\mathrm{mAb}$ against VE-cadherin (BV13). Remarkably, the combination of CA4P and anti-VE-cadherin resulted in hemorrhage within the tumors, demonstrating the disruption of the endothelial barrier function, which is usually mediated through VE-cadherin engagement (Figure 5B, arrowhead).

To gain insight into the mechanism by which $\mathrm{CA} 4 \mathrm{P}$ or $\mathrm{mAb}$ against VE-cadherin (BV13) induced tumor necrosis, we investi- gated the effect of such treatments on tumor cell death by TUNEL assay. The tumor sections obtained from the untreated mice were negative for TUNEL assay (Figure 5C). In sharp contrast, large areas of tumor mass were necrotic in groups of mice treated with CA4P or mAb against VE-cadherin (BV13) and to a much higher degree in groups of mice treated with a combination of CA4P and $\mathrm{mAb}$ against VE-cadherin (BV13) (Figure 5C, arrows).

To investigate the effect of CA4P on tumor angiogenesis, the extent of intratumoral vascularization within the different groups was assessed by CD31 (PECAM-1) immunostaining of tumor sections. The control group exhibited abundant number of vessels within the tumor as compared with $\mathrm{CA} 4 \mathrm{P}$ or $\mathrm{mAb}$ against VEcadherin (BV13) or CA4P plus mAb against VE-cadherin (BV13) groups (Figure 6A). Indeed, microvessel density determination showed that both CA4P- and anti-VE-cadherin-treated mice developed a limited number of neovessels within the tumors as compared with that observed in the control group $(13.75 \pm 1.37$ and $14.0 \pm 2$ versus $28.75 \pm 4.62$ positive cells per field; $P<0.05 ; n=5$; Figure 6B). The most remarkable result was obtained after CA4P plus anti-VE-cadherin treatment. Evaluation of the microvessel 
A

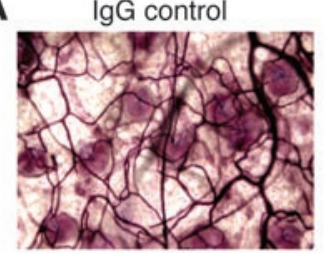

Anti-VE-

cadherin (BV13)

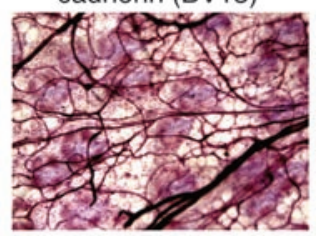

Anti-VE.

cadherin (E4G10)

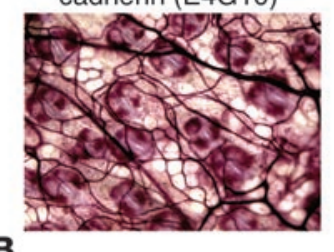

$\mathbf{B}$

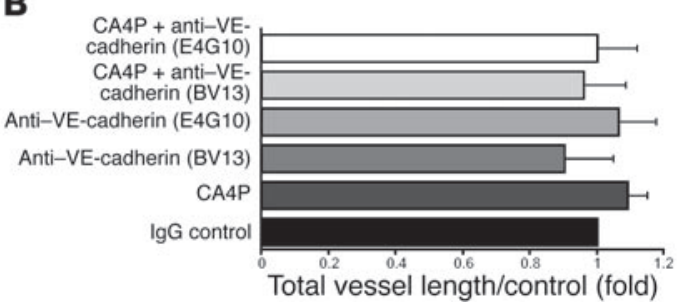

Figure 8

CA4P used alone or in combination with $\mathrm{mAb}$ against VE-cadherin does not destabilize normal vasculature. (A) CA4P does not affect normal vasculature. Mouse ears were injected with CA4P $(100 \mu \mathrm{g})$, mAb against VE-cadherin BV13 $(10 \mu \mathrm{g})$, mAb against VE-cadherin E4G10 $(150 \mu \mathrm{g})$, or a mix of CA4P plus mAb against VE-cadherin, and the control group received $150 \mu \mathrm{g}$ of IgG antibody. Seven days after injection, ears were removed and stained for vessels using PECAM-1 staining. VIP-based immunodetection yielded a red reaction product. Magnification: $\times 20$. (B) Quantitative analysis of the total vessel length in the mouse ears. Total vessel length was obtained from 5 peripheral fields for each ear of each animal ( $n=5 /$ group). Results show the total vessel length relative to that of control.

density indicated a marked reduction in the number of microvessels after combined CA4P plus anti-VE-cadherin (BV13) treatment as compared with the control group $(5 \pm 1.5$ versus $28.75 \pm 4.62$ positive cells per field, respectively; $P<0.001 ; n=5$; Figure 6B). The fact that neutralizing $\mathrm{mAb}$ against VE-cadherin remarkably enhanced the CA4P effect suggests that CA4P exerts its antivascular effect in part through destabilization of VE-cadherin, which is essential for the integrity of the angiogenic endothelial cells.

Although clinically CA4P has been typically used for short periods of time, we examined the effect of chronic treatment with CA4P beyond 8 days on tumor microvessel density. The number of capillaries and those capillaries that were associated with SMCs was assessed by costaining with PECAM- 1 and $\alpha$-SMA. Remarkably, although the tumors isolated from the untreated groups showed significant SMC-invested capillaries, a few areas of staining for $\alpha$-SMA were detected in the CA4P and the CA4P plus anti-VE-cadherin (BV13) groups (Figure 6C). Quantification of SMC-ensheathed neovessels indicated a marked reduction in the number of ensheathed neovessels after CA4P and CA4P plus anti-VE-cadherin treatment as compared with control group $(11.75 \% \pm 5.75 \%$ and $10.75 \% \pm 2.75 \%$ versus $57.5 \% \pm 11 \%$ SMCensheathed neovessels per field, respectively; $P<0.001 ; n=5$; Figure 6D). These data suggest that chronic treatment with CA4P diminishes the total number of capillaries and those capillaries that are ensheathed with SMCs. Taken together, these findings suggest that chronic exposure to CA4P not only targets unstable vessels, but also induces regression of the SMC-coated neovessels that may undergo remodeling. It is possible that during the remodeling process, the endothelial cells may transiently become disengaged from SMCs, rendering them sensitive to CA4P. As such, CA4P may also play a role in pruning and sculpting unstable neovessels during the remodeling process.

The neutralizing mAb against VE-cadherin (BV13) used in previous experiments is highly effective in disrupting the interaction of fully engaged VE-cadherin within the established stable neovessels. However, several lines of evidence have shown that subsets of tumor vasculature are leaky due to partial disengagement of VE-cadherin. Hence, these nascent vessels may be exclusively sensitive to CA4P and susceptible to complete disruption of VE-cadherin interaction. To test this hypothesis, we took advantage of the availability of the neutralizing $\mathrm{mAb}$ against VEcadherin (clone E4G10) that binds to an epitope of VE-cadherin that is only exposed in partially or fully disengaged leaky tumor endothelial cells $(9,30)$. CA4P also synergized with this $\mathrm{mAb}$ against VE-cadherin (E4G10) to block tumor growth $(P<0.001$; $n=9$; Figure $7 \mathrm{~A}$ ). These results were similar to those obtained with a combination of CA4P and BV13. Histological examination of tumor sections showed areas of necrosis in groups of mice that were treated with either CA4P or mAb against VE-cadherin (E4G10) (Figure 7B, arrows), with much higher areas of necrosis when a combination of anti-VE-cadherin (E4G10) and $\mathrm{CA} 4 \mathrm{P}$ was used. However, CA4P used in combination with $\mathrm{mAb}$ against VE-cadherin (E4G10) was less effective than BV13 in promoting tumor hemorrhage (Figure 7B, arrowhead). Nonetheless, combined treatment with CA4P and mAb against VE-cadherin (E4G10) showed a synergistic effect in blocking tumor neoangiogenesis $(P<0.05 ; n=5$; Figure $7, \mathrm{C}$ and $\mathrm{D})$.

Finally, the effects of the different treatments using CA4P alone or in combination with $\mathrm{mAb}$ against VE-cadherin (BV13 or E4G10) were assessed on normal vasculature using the ear model of angiogenesis in mice (31). CA4P used alone or in combination with E4G10 or therapeutic nontoxic doses of BV13 did not target the normal vessels, as demonstrated by PECAM-1 staining of the vessels (Figure $8 \mathrm{~A}$ ) and confirmed by the quantification of the total length of the vessels per field as compared with the IgG control (Figure 8B). Collectively, these data suggest that partially disengaged endothelial cells are more susceptible to the VE-cadherin destabilizing agents, resulting in selective targeting of leaky tumor neovessels. Since preclinical use of E4G10 has been associated with very low vascular toxicity as compared with BV13, the combination of E4G10 and CA4P will provide a highly effective means to target nascent unstable tumor vasculature.

\section{Discussion}

Vascular disrupting agents were developed to induce selective regression of atypical tumor blood neovessels (16-18). CA4P, as 


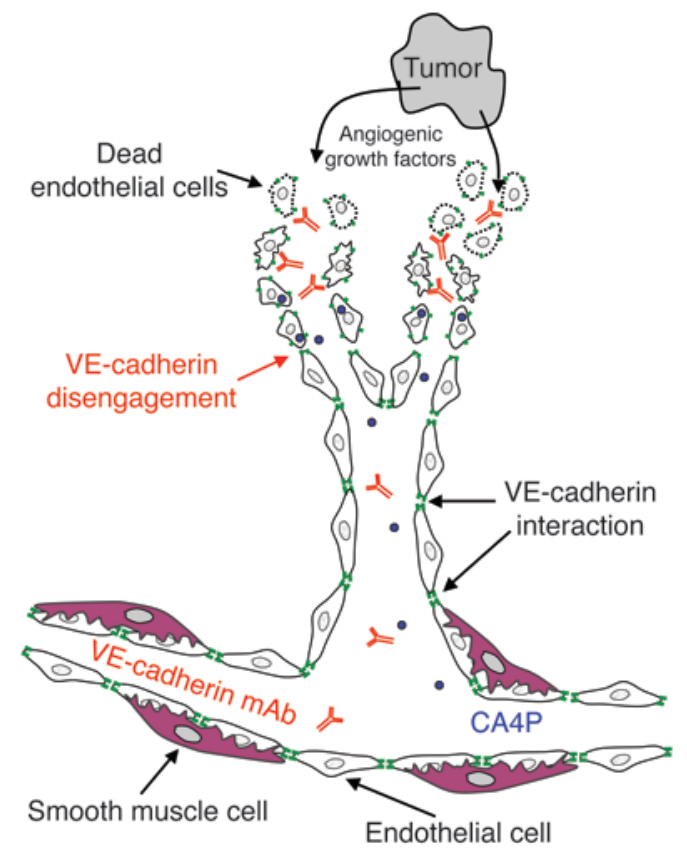

Figure 9

Proposed model for CA4P-mediated angiogenesis inhibition. Preexisting vessels are invested with SMCs protecting endothelial cells against CA4P-induced cell death. Because nascent unstable tumor neovessels are not ensheathed by periendothelial mural cells, CA4P selectively destabilizes neovessels by inducing VE-cadherin disengagement, therefore increasing the antiangiogenic effects of the neutralizing $\mathrm{mAb}$ against VE-cadherin without increasing toxicity to the normal vasculature.

a prototypical microtubule-destabilizing agent, exerts its antivascular effect presumably by taking advantage of the relative instability of tumor vasculature and its supporting structures, thereby inducing a rapid collapse and regression of tumor vessels, leading to ischemic or hemorrhagic necrosis. However, the molecular mechanisms by which CA4P exerts its selective antitumor vascular effects are not fully unraveled. Here, we show that CA4P selectively targets unstable tumor neovessels through rapid disengagement of the VE-cadherin/ $\beta$-catenin complex, destabilization of cytoskeletal organization of endothelial cells, and subsequent non-apoptotis-mediated cell death. Inhibition of VE-cadherin homotypic interaction synergized with the microtubule-destabilizing effect of CA4P, resulting in regression of preestablished tumor neovessels and diminished tumor growth. In addition, we show that stabilization of endothelial cells by investment with SMCs confers significant resistance to CA4P. These data suggest that nascent unstable tumor neovessels, lacking proper periendothelial support, are sensitive to the microtubule-destabilizing effects of CA4P through VE-cadherin disengagement.

By disrupting VE-cadherin/ $\beta$-catenin complex, CA4P inhibits various endothelial cell vital functions. One remarkable effect of CA4P on endothelial cells is the induction of cell death at very low nontoxic doses. However, CA4P does not induce endothelial cell death through known apoptotic pathways. In fact, exposure of endothelial cells to CA4P initiates a series of profound morphological alterations that affect the integrity of cell shape causing anchorage loss, thereby impairing survival signals, and ultimately leading to cell death, features that are reminiscent of anoikis- mediated cell death. Indeed, anoikis refers to cell death induced by a loss of cell-matrix interactions and/or through dysregulation of cell-cell interactions and controls cell survival, particularly via cadherin homophilic ligation (32). This unique, rapid induction of the endothelial cell death may explain the immediate induction of vascular collapse and tumor necrosis imparted by CA4P upon certain tumors such as anaplastic thyroid cancer.

Concomitant with inhibition of endothelial cell proliferation, a significant inhibitory effect of CA4P on endothelial cell migration was demonstrated using a wound assay. A three-dimensional tube formation assay revealed that CA4P blocks formation of a capillary tube network. In addition, CA4P can destabilize a preestablished endothelial network, disrupting endothelial cell lining, resulting in cell rounding. In fact, endothelial cells undergoing remodeling or participating in neovessel assembly are in a dynamic state during angiogenesis and are thus not firmly attached to the extracellular matrix or to periendothelial cells, such as SMCs or pericytes. This makes them more vulnerable to anoikis-mediated cell death, a process promoted by loss of cell-matrix and/ or cell-cell attachment (32). It seems that an early event in the cytotoxic process triggered by CA4P on endothelial cells is mediated through alterations of cell-cell interactions. In fact, whereas angiogenic endothelial cells are characterized by a flattened morphology and by functional adherens junctions between other endothelial cells, CA4P-treated endothelial cells retracted their cytoplasm, became round, and lost contact with neighboring cells. In contrast, endothelial cells when cocultured with SMCs were stabilized and were not sensitive to CA4P. To the same extent, vascular tube formation was also preserved in the presence of SMCs. These findings suggest that endothelial cells are able to overcome cell death by anoikis when VE-cadherin mediated cell-cell contact is preserved in the presence of SMCs.

CA4P not only inhibits vascular endothelial cell tube formation, but also disrupts endothelial cell-cell contacts, preventing establishment of cellular connections that are critical for their survival and morphological integrity. These effects are in part attributed to the inhibition of the function of the VE-cadherin/ $\beta$-catenin complex in vascular endothelial cells. Indeed, CA4P induced the rapid redistribution of $\mathrm{VE}$-cadherin/ $\beta$-catenin complex along the adjacent cells before the initiation of cellular retraction. CA4P had no major apparent effect on the integrity and maintenance of other junctional proteins including ZO-1 and occludin, PECAM-1, and connexin-43, although a possible destabilizing effect of other as-yet-unrecognized junctional proteins by CA4P can not be completely excluded. Collectively, these data suggest that CA4P primarily targets unstable tumor neovessels by selective and efficient disruption of endothelial-specific VE-cadherin. In support of this conclusion, we show that CA4P rapidly diminished the tyrosine phosphorylation of VE-cadherin and $\beta$-catenin, thereby interfering with the maintenance of a functional endothelial cell barrier. This result may not fit with the VEGF-A-induced tyrosine phosphorylation of VE-cadherin, which has been reported to be an important regulatory pathway associated with endothelial barrier dysfunction and increased permeability (33). Our unique finding, which was obtained in the absence of VEGF-A stimulation, may be a characteristic of microtubule-destabilizing agents, and the precise mechanism of activation of the intracellular events leading to the tyrosine phosphorylation of the VE-cadherin/ $\beta$-catenin complex is the subject of ongoing studies. Nonetheless, CA4P remarkably triggered serine/threonine phosphorylation of 
$\beta$-catenin, which targets $\beta$-catenin for ubiquitination and proteasomal degradation $(28,29)$.

To formally prove that the stability of VE-cadherin/ $\beta$-catenin complex is critical for the antivascular effect afforded by CA4P, we show that infection of endothelial cells with AdNullE4 $4^{+}$vectors, which activate and stabilize VE-cadherin homotypic interaction, confers a robust resistance to CA4P (27). Remarkably, cotreatment of AdNulle4+-infected endothelial cells with CA4P and neutralizing $\mathrm{mAb}$ against $\mathrm{VE}$-cadherin decreased endothelial cell viability and also impaired endothelial cell barrier function, bolstering the notion that CA4P exerts its effect in part through VE-cadherin disengagement. In addition, neutralizing $\mathrm{mAb}$ against $\mathrm{VE}$-cadherin not only inhibited new capillary formation, but also induced the disruption of mature vessels (7). In fact, CA4P induces disruption of actin filaments, and this effect can result in endothelial cell detachment by disrupting the intracellular organization of the cytoskeleton, contributing to physical VE-cadherin disengagement. VE-cadherin and $\beta$-catenin chemically cross-link with PI3K and support the survival of endothelial cells and the development of new capillaries in a converging Akt signaling pathway (4). The fact that CA4P decreased the phosphorylation of Akt supports the concept that VE-cadherin/ß-catenin inhibition and PI3K/Akt blockade by CA4P blocks tumor neovessel assembly.

Treatment of tumor-bearing mice with high doses of neutralizing $\mathrm{mAb}$ against murine $\mathrm{VE}$-cadherin (BV13) has been shown to inhibit angiogenesis and tumor growth $(8,9)$. However, preclinical use of $\mathrm{mAb}$ against VE-cadherin (BV13) has been associated with significant increase in vascular permeability and toxicity, because BV13 binds to an epitope on the amino-terminal loops of VE-cadherin that is readily accessible even in fully stabilized, established vessels, thereby tempering the enthusiasm for clinical use. In our study, we used lower nontoxic doses of mAb against VE-cadherin (BV13), which blocked tumor neoangiogenesis to the same degree as did treatment of mice with CA4P without significant toxicity. Cotreatment with CA4P and mAb against VE-cadherin (BV13) resulted in a significant inhibition of tumor neoangiogenesis with minimal toxicity. Histological examination of tumors removed from animals after treatment showed dramatic differences between the groups treated with CA4P, mAb against VE-cadherin (BV13), or CA4P plus mAb against VE-cadherin (BV13) and the control group. Indeed, tumors from animals receiving either CA4P or anti-VE-cadherin (BV13) or a combination CA4P plus anti-VEcadherin (BV13) treatment showed enhanced tumor necrosis.

Similar results were obtained using another $\mathrm{mAb}$ against antiVE-cadherin (E4G10), which can bind only to the endothelial cells, where VE-cadherin is partially engaged $(9,30)$. These findings suggest that CA4P may selectively destabilize leaky tumor endothelial cells, thereby exposing hidden VE-cadherin epitopes to E4G10. Thus, the synergistic effects of CA4P with $\mathrm{mAb}$ against VE-cadherin (E4G10) account for a selective targeting of unstable tumor neovessels by CA4P without conferring vascular toxicity to other stabilized organ vasculature.

Moreover, costaining of vessels and SMCs showed that chronic treatment with CA4P for 8 days resulted in the regression of both neovessels and SMC-coated vessels. However, the mechanisms by which CA4P might impair SMC investment of neovessels remain unclear. A possible indirect reduction of SMCs after CA4P treatment through dismantling of vascular structures cannot be excluded. Nevertheless, whatever the effects induced by chronic treatment of CA4P to block SMC investment of neovessels, lack of SMC ensheathment of the tumor neovessels may make these immature capillaries susceptible to further disengagement of VEcadherin afforded by introduction of CA4P or low doses of $\mathrm{mAb}$ against VE-cadherin. This may also explain the specificity of CA4P in targeting certain nascent tumor neovessels, since these immature vessels lack supportive mural cells, while subsequent acquisition of mural cell coverage leads to vessel stabilization, thereby conferring resistance to CA4P (Figure 9).

One major clinical complication associated with the use of CA4P may be the impairment of wound healing or collateral vessel formation after myocardial or cerebral infarction. Our data suggest that fully stabilized vessels, such as the vasculature in the mouse ears, are resistant to CA4P and low doses of mAb against VE-cadherin. However, anecdotal reports have attributed vascular toxicity of CA4P to disruption of myocardial ischemic neovessels. The requirement for using high doses and potentially toxic doses of CA4P may be alleviated by combining lower and less toxic doses of CA4P with lower doses of anti-VE-cadherin agents. Nonetheless, additional rigorous studies are in order to assess whether a combination of lower doses of CA4P and anti-VE-cadherin (i.e., E4G10) are going to be any less toxic than the use of higher doses of either agent.

Recent evidence suggests that antiangiogenic agents may exert their antitumor effect by "normalizing" tumor vasculature (34, 35). Normalization of tumor blood vessels is associated with pruning of unstable, leaky, and pericyte-depleted tumor vessels that are susceptible to the antiangiogenic agents, including anti-VEGF-A and anti-VEGFR-2. This normalization process, which takes several days and results in removal of unstable tumor neovessels and maintenance of fully stabilized tumor vessels, provides a conduit to efficiently deliver chemotherapeutic agents to the tumor cells. Similar to this process, it is plausible that CA4P may induce rapid pruning and sculpting of unstable tumor neovessels, thereby normalizing tumor neovessels. As such, combination of CA4P will also increase the delivery of chemotherapeutic agents to the tumor cells, thereby enhancing their therapeutic index.

Taken together, our data suggest that 1 mechanism by which CA4P exerts its antiangiogenic effect is through rapid functional inhibition of the VE-cadherin/ $\beta$-catenin complex as needed for endothelial cellcell adhesion and survival during neovessel assembly and remodeling. CA4P may also inhibit angiogenesis by Akt inactivation, which could interfere with endothelial cell proliferation, gene expression, and tube formation. These findings set forth a novel mechanistic insight into the potential effects of CA4P on the reduction of neoangiogenesis and tumor growth. These data also provide justification for using antiangiogenic agents, such as anti-VE-cadherin, in conjunction with antivascular agents and chemotherapeutic agents to effectively block tumor angiogenesis and diminish end-organ vascular toxicity. Indeed, as anti-VE-cadherin treatment is associated with significant toxicity, combined use of antivascular agents in conjunction with lower doses of anti-VE-cadherin may diminish toxicity and improve the efficacy of the treatment.

\section{Methods}

\section{Reagents}

R\&D Systems supplied recombinant human VEGF-A and FGF-2. When HUVECs were incubated with FGF-2, 1\% heparin (Sigma-Aldrich) was systematically added in the cell culture medium. CA4P was dissolved in PBS to $0.01 \mathrm{M}$ and stored at $4^{\circ} \mathrm{C}$. Further dilutions to working concentrations were made before use. CA4P was kindly provided by OXiGENE Inc. 


\section{Cell culture}

HUVECs and SMCs were isolated from umbilical cord veins with collagenase and were cultured in M199 medium containing 10\% (vol/vol) FBS, $20 \mu \mathrm{g} / \mathrm{ml}$ endothelial cell growth factor, $50 \mu \mathrm{g} / \mathrm{ml}$ heparin, $100 \mu \mathrm{g} / \mathrm{ml}$ penicillin, and $100 \mu \mathrm{g} / \mathrm{ml}$ streptomycin in a humidified incubator at $37^{\circ} \mathrm{C}$ with air $/ 5 \% \mathrm{CO}_{2}$. HUVEC and SMC monolayers from passages $2-4$ were used in these studies.

\section{Cell proliferation assay}

For the proliferation assay, we used the minimal concentration of FBS (1\%) diluted in X-VIVO medium (Cambrex Corp.) to allow sufficient viability of endothelial cells. Briefly, after detachment, the cells were seeded at a concentration of $2 \times 10^{4} \mathrm{HUVEC}$ in each well of 24 -well plates, allowed to adhere overnight, and then incubated with or without cytokines $(5 \mathrm{ng} / \mathrm{ml}$ FGF-2 or $5 \mathrm{ng} / \mathrm{ml}$ VEGF-A). CA4P was added at concentrations indicated in Results. After incubation for 12, 24, 36, and 48 hours, cells were detached by trypsin/EDTA and manually counted using trypan blue exclusion. For the coculture experiment, HUVECs and SMCs were stained using red fluorescent cell linker PKH26 and green fluorescent cell linker PKH2, respectively, according to the manufacturer's instructions (Sigma-Aldrich), and $2.5 \times 10^{4}$ HUVECs and $2.5 \times 10^{4}$ SMCs were seeded together in each well of 24-well plates in X-VIVO supplemented with 5\% FBS. Cells were then differentially counted under a fluorescent microscope. Each fluorescent cell was checked for the presence of trypan blue by switching to white light.

\section{Apoptosis analysis}

FGF-2-stimulated endothelial cells were treated with CA4P $(0,10$, and $50 \mathrm{nM})$ for 12, 24, or 36 hours and analyzed for the presence of apoptotic cells using the ApoAlert Annexin V-FITC PI Apoptosis Kit (BD), following the manufacturer's instructions. Apoptotic analysis was performed using a Beckman-Coulter Elite Flow Cytometer.

\section{Cell wounding}

HUVECs $\left(1.5 \times 10^{5}\right.$ per well) were seeded in 6 -well plates and incubated at $37^{\circ} \mathrm{C}$ until they attached. A single lesion of $1 \mathrm{~mm}$ in width was produced across the cell monolayer by mechanical scraping of the adherent endothelial cells. Wells were rinsed with PBS to remove dislodged cells and cellular debris, and unstimulated or FGF-2-stimulated cells were incubated in X-VIVO supplemented with 1\% FBS (minimum serum content required for wounded monolayers to remain attached to the wells during the incubation period without inducing proliferation). After 24 hours, wells were washed 3 times with PBS and fixed in 4\% paraformaldehyde (PFA) for 10 minutes prior to image analysis. Recovery of each denuded area was quantified by counting any single cells or small groups of cells that moved into 5 fields of view taken at random.

\section{Capillary tube formation on a Matrigel matrix}

Matrigel matrix (BD) was kept on ice for 24 hours. Then, $200 \mu \mathrm{l}$ per well of Matrigel was added to 24 -well culture plates. After gelation at $37^{\circ} \mathrm{C}$ for 30 minutes, gels were overlaid with $500 \mu$ l of medium containing $3 \times 10^{4}$ HUVECs. Afterward, endothelial cells were stimulated with $5 \mathrm{ng} / \mathrm{ml}$ of FGF-2 and then incubated with different concentrations of CA4P as indicated in Results. The effect of CA4P on capillary tube formation was inspected after a period of 12 hours under an inverted light microscope. The quantification of the capillary tube formation was performed by measuring the tubule length and counting the number of branch points in 4 different pictures taken at random. For tube formation in the presence of SMCs, HUVECs $\left(3 \times 10^{4}\right)$ stained in red fluorescent cell linker PKH26 (Sigma-Aldrich) were seeded with SMCs $\left(1 \times 10^{4}\right)$ stained with green fluorescent cell linker PKH2 (Sigma-Aldrich) on Matrigel matrix, and the effect of CA4P was analyzed after a 12-hour incubation under an inverted fluorescent microscope. In another experiment, CA4P was added on a preestablished FGF-2-stimulated capillary network (after a 3-hour incubation with angiogenic growth factor), and the effect of CA4P on the disruption of the capillary network was monitored every 3 hours.

\section{Cell shape observation}

HUVECs were allowed to grow until they reached $80 \%$ confluence. HUVECs were then stimulated with FGF-2 and incubated with CA4P in X-VIVO supplemented with $5 \%$ FBS, and modification of the cell shape was monitored at different time points under an inverted microscope.

\section{Fluorescence microscopy analysis}

The fluorescence microscopy analysis of VE-cadherin, $\beta$-catenin, phospho$\beta$-catenin Ser33/37/Thr41, CD31, ZO-1, occludin, connexin- 43 , and actin filaments was performed on the FGF-2-stimulated HUVECs in different conditions and times, as indicated in Results. Briefly, after HUVECs were fixed in 4\% PFA and permeabilized with $0.1 \%$ Triton X-100, HUVECs were incubated with polyclonal antibodies against VE-cadherin (1:100; Alexis Biochemicals), $\beta$-catenin (1:100; Santa Cruz Biotechnology Inc.), phospho- $\beta$-catenin Ser33/37/Thr41 (1:100; Cell Signaling Technology), ZO-1 (1:100; Zymed Laboratories Inc.), occludin (1:20; Zymed Laboratories Inc.), connexin-43 (1:100; Zymed Laboratories Inc.), and mAb against PECAM-1 (1:50; Alexis Biochemicals) for 1 hour at room temperature, then washed and incubated with secondary Texas red-conjugated antibodies (1:1000; Vector Laboratories) for 1 hour at room temperature. After washing, the samples were mounted in VECTASHIELD (Vector Laboratories) and analyzed by fluorescence microscopy at $\times 40$ magnification (Olympus). Changes in actin organization were investigated with TRITC-labeled phalloidin (Sigma-Aldrich) diluted at $4 \mu \mathrm{g} / \mathrm{ml}$ in PBS, which selectively stains F-actin and does not bind to monomeric G-actin. After fixation and permeabilization, cells were incubated for 1 hour at room temperature with phalloidin, rinsed with PBS, and observed with an inverted fluorescent microscope.

\section{Infection of HUVECs with AdNullE4 vector}

HUVEC monolayers were seeded in 12-well plates, allowed to adhere, and infected with AdNullE4- or AdNullE4+ overnight in serum-free medium as described previously (27). Cells were then incubated with CA4P or human anti-VE-cadherin $\mathrm{mAb}$ (BV9; ImClone Systems Inc.) or in combination at concentrations indicated in Results. Cell viability was assayed by trypan blue exclusion 48 hours after the treatment.

\section{Endothelial cell permeability assay}

AdNullE4-- or AdNullE4+-infected HUVECs were seeded at $2.5 \times 10^{5}$ cells on Transwell filters ( $3 \mu \mathrm{m}$ pore size, $6.5 \mathrm{~mm}$ diameter; Corning Inc.) precoated with collagen (StemCell Technologies) according to the manufacturer's instructions, and cultured for 72 hours. HUVECs were then incubated with either CA4P or neutralizing mAb against VE-cadherin (BV9) or in combination. Modification of endothelial cell monolayer permeability was monitored at different time points by adding FITC-labeled dextran 3.3-kDa (10 $\mu \mathrm{g} / \mathrm{ml}$; Invitrogen Corp.) to each Transwell insert for 1 hour, followed by fluorescence measurement of medium from the lower well on the SpectraMax M2 microplate reader (Molecular Devices) using excitation/emission wavelengths of 494/521 nm.

\section{Western blot analysis}

HUVECs $\left(3 \times 10^{6}\right)$ were incubated under different conditions and for various times as indicated in Results after an overnight starvation with X-VIVO medium. Western blot cell extracts were then prepared by lysing cells in cold RIPA buffer (50 mM Tris, 1\% NP-40, 0.15\% Na-deoxycholate, $150 \mathrm{mM} \mathrm{NaCl}, 1 \mathrm{mM}$ EDTA, $0.1 \%$ SDS) in the presence of protease inhibitors (aprotinin, leupeptin, pepstatin, and phenylmethylsulfonyl fluoride). After sonication and centrifu- 
gation to remove cell debris, the protein yield was quantified using the BCA protein assay kit (Pierce Biotechnology Inc.). Normalized cell lysates were then mixed with sample buffer containing 2-mercaptoethanol and SDS and heated for 5 minutes at $95^{\circ} \mathrm{C}$. Samples containing $50 \mu \mathrm{g}$ total proteins were run on SDS-polyacrylamide gels before being transferred to nitrocellulose membranes. Binding of the primary antibody against polyclonal Akt or polyclonal Akt phospho-Ser473 (1:1,000; Cell Signaling Technology), polyclonal $\beta$-catenin phospho-Ser45 (1:1,000; Cell Signaling Technology), polyclonal $\beta$-catenin phospho-Ser33/37/Thr41 (1:1,000; Cell Signaling Technology), polyclonal $\beta$-catenin (1:500; Santa Cruz Biotechnology Inc.), and polyclonal VE-cadherin (1:500; Santa Cruz Biotechnology Inc.) was detected with the enhanced chemiluminescence reagent (Amersham Pharmacia Biotech) using HRP-conjugated secondary antibody (1:5,000; DakoCytomation).

\section{Immunoprecipitation of VE-cadherin and $\beta$-catenin and phosphotyrosine Western blotting}

HUVECs $\left(3 \times 10^{6}\right)$ were incubated in different conditions and times as indicated in Results after an overnight starvation with X-VIVO medium. Cells were lysed in $0.5 \mathrm{ml}$ ice-cold RIPA buffer containing protease inhibitors and $1 \mathrm{mM} \mathrm{Na}_{3} \mathrm{VO}_{4}$. The protein concentration of the cell lysate was then determined, and goat antibody against VE-cadherin ( $4 \mu \mathrm{g}$; Santa Cruz Biotechnology Inc.) or rabbit antibody against $\beta$-catenin ( $3 \mu \mathrm{g}$; Santa Cruz Biotechnology Inc.) was added to the same amount of protein lysate and incubated for 2 hours at $4^{\circ} \mathrm{C}$. The immunocomplex was captured by $100 \mu$ l protein $\mathrm{G}$ agarose beads (Santa Cruz Biotechnology Inc.) for 1 hour, and then beads were sedimented by brief centrifugation and washed by resuspending and pelleting 3 times with $0.5 \mathrm{ml}$ ice-cold modified RIPA buffer. Then, the agarose beads were resuspended in $60 \mu \mathrm{l}$ sample buffer, boiled for 5 minutes, and collected by centrifugation, and $20 \mu \mathrm{l}$ of the supernatant fraction was fractionated on $7.5 \%$ SDS-polyacrylamide gel and processed for immunoblotting with antiphosphotyrosine antibody (1:200; Santa Cruz Biotechnology Inc.).

\section{In vivo tumorigenesis assay}

Tumor model in mice. Approval for performing these experiments was obtained from the institutional review board at Weill Medical College of Cornell University. B16 melanoma cells were obtained from ATCC and were cultured in DMEM (Invitrogen Corp.), supplemented with 10\% FBS, and antibiotics. B16 cells were harvested, washed, and resuspended in XVIVO, and a volume of $200 \mu \mathrm{l}$ containing $2 \times 10^{6}$ cells was injected subcutaneously into the dorsa of 7-week-old female C57BL/6 mice (Jackson Laboratory) using a 24-gauge needle. When mice bore a tumor of approximately $200 \mathrm{~mm}^{3}$ (i.e., after 10 days), 4 experimental groups were randomized, each with 9 animals. Treatment was initiated at this time and continued every 2 days. The CA4P group was subjected to intravenous injection of CA4P at $5 \mathrm{mg} / \mathrm{kg}$ body weight; the anti-VE-cadherin group received intraperitoneal injection of $10 \mu \mathrm{g}$ of monoclonal anti-VE-cadherin antibody (mAb BV13) or $1 \mathrm{mg}$ of monoclonal anti-VE-cadherin antibody (mAb E4G10) directed against mouse VE-cadherin; the combined group received CA4P $(5 \mathrm{mg} / \mathrm{kg})$ plus monoclonal anti-VE-cadherin antibody $(10 \mu \mathrm{g}$ of BV13 or $1 \mathrm{mg}$ of $\mathrm{E} 4 \mathrm{G} 10$ ); and the control group received $10 \mu \mathrm{g}$ of $\operatorname{IgG}$ (6). After different times, tumor appearance was evaluated using a caliper, and tumor volume was calculated using the formula $\pi / 6\left(w_{1} \times w_{2} \times w_{2}\right)$, where $w_{1}$ represents the largest tumor diameter, and $w_{2}$ represents the smallest tumor diameter. After an 8-day treatment, animals were sacrificed, and tumors were removed and then subjected to immunohistochemistry analysis. Some tumors were embedded in Tissue-Tek OCT (VWR International) com- pound and immediately frozen in liquid nitrogen; others were embedded in paraffin. Paraffin-embedded tumors were serially sectioned and stained with $\mathrm{H} \& \mathrm{E}$ for histological analysis.

TUNEL staining. Cell death within paraffin-embedded tumor sections was detected by TUNEL reaction (Roche Diagnostics $\mathrm{GmbH}$ ) according to the manufacturer's instructions. Assessment of cell death in this assay relies on the detection of free $3^{\prime} \mathrm{OH}$ DNA ends. Positive signal was revealed by fast red staining, and tumor sections were then counterstained prior to analysis by light microscope.

Immunohistochemical staining. Immunohistochemistry was used to assess the extent of intratumoral vascularization and SMC investment within the different experimental groups. Five-micrometer frozen tumor sections were prepared. For vessel staining, endogenous peroxidase was quenched using $3 \%$ aqueous $\mathrm{H}_{2} \mathrm{O}_{2}$, and sections were incubated overnight at $4{ }^{\circ} \mathrm{C}$ with a biotinylated rat antibody against mouse PECAM-1 (1:100; BD Biosciences - Pharmingen) and then with Rhodamine Avidin DCS (1:500; Vector Laboratories) for 1 hour at room temperature. SMCs were detected using a rabbit antibody against $\alpha$-SMA (1:100; Abcam Inc.) and then secondary FITC-conjugated antibodies (1:1,000; Vector Laboratories). After washing, the sections were then mounted in VECTASHIELD containing DAPI (Vector Laboratories) and analyzed by fluorescence microscopy. The microvessel density and the number of SMC-ensheathed vessels were evaluated by microscopic counting of 5 fields at $\times 10$ magnification and are presented as mean number of microvessels and SMC-ensheathed vessels per microscopic field.

\section{Ear model}

The effect of CA4P alone or used in combination with the different anti-VEcadherin antibodies (BV13 or E4G10) was investigated on the normal vasculature using the mouse ear model as previously described (31). Each group contained 5 animals, and injections of CA4P $(100 \mu \mathrm{g})$ in the presence or absence of monoclonal anti-VE-cadherin antibodies $(10 \mu \mathrm{g}$ BV13 and $150 \mu \mathrm{g}$ E4G10) were performed bilaterally. The control group received $150 \mu \mathrm{g}$ IgG. Animals were sacrificed 7 days after injection. A minimum of 5 regions of ear skin were photographed at the distal periphery, and blood vessels were quantitated by measuring the total length of vessels lined by PECAM-1positive endothelial cells using Photoshop Image version 7.0 (Adobe Systems Inc.) analysis. All analyses were performed in a blinded fashion.

\section{Statistics}

Both in vitro and in vivo results were statistically analyzed using a 2-tailed nonparametric Mann-Whitney $U$ test. The results are expressed as mean value \pm SEM. $P<0.05$ was considered significant.

\section{Acknowledgments}

This work was supported by grants from the American Cancer Society, the NIH (RO1-HL075234, HL59312, HL66592, HL61849, and HL67839), and the Howard Hughes Medical Institute.

Received for publication January 27, 2005, and accepted in revised form July 26, 2005.

Address correspondence to: Shahin Rafii or Loïc Vincent, Weill Medical College of Cornell University, 1300 York Avenue, Room A-863, New York, New York 10021, USA. Phone: (917) 287-3801; Fax: (212) 746-8481; E-mail: srafii@med.cornell.edu (S. Rafii) or vincentloicny@yahoo.com (L.Vincent).
1. Zhu, Z., et al. 2003. Inhibition of human leukemia in an animal model with human antibodies directed against vascular endothelial growth factor receptor 2. Correlation between antibody affinity and biological activity. Lenkemia. 17:604-611.

2. Dejana, E. 1996. Endothelial adherens junctions: implications in the control of vascular permeability and angiogenesis. J. Clin. Invest. 98:1949-1953.
3. Breviario, F., et al. 1995. Functional properties of human vascular endothelial cadherin (7B4/ cadherin-5), an endothelium-specific cadherin. Arterioscler. Thromb. Vasc. Biol. 15:1229-1239. 
4. Carmeliet, P., et al. 1999. Targeted deficiency or cytosolic truncation of the VE-cadherin gene in mice impairs VEGF-mediated endothelial survival and angiogenesis. Cell. 98:147-157.

5. Caveda, L., et al. 1996. Inhibition of cultured cell growth by vascular endothelial cadherin (cadherin5/VE-cadherin). J. Clin. Invest. 98:886-893.

6. Corada, M., et al. 1999. Vascular endothelial-cadherin is an important determinant of microvascular integrity in vivo. Proc. Natl. Acad. Sci. U. S. A. 96:9815-9820

7. Bach, T.L., et al. 1998. VE-cadherin mediates endothelial cell capillary tube formation in fibrin and collagen gels. Exp. Cell Res. 238:324-334.

8. Liao, F., et al. 2000. Monoclonal antibody to VE-cadherin is a potent inhibitor to angiogenesis, tumor growth, and metastasis. Cancer Res. 60:6805-6812.

9. Liao, F., et al. 2002. Selective targeting of angiogenic tumor vasculature by vascular endothelialcadherin antibody inhibits tumor growth without affecting vascular permeability. Cancer Res. 62:2567-2575.

10. Carmeliet, P. 2000. Mechanisms of angiogenesis and arteriogenesis. Nat. Med. 6:389-395.

11. Yancopoulos, G.D., et al. 2000. Vascular-specific growth factors and blood vessel formation. Nature. 407:242-248.

12. Jain, R.K., and Carmeliet, P. 2001. Vessels of death or life. Sci. Am. 285:38-45.

13. Folkman, J. 1995. Angiogenesis in cancer, vascular, rheumatoid and other diseases. Nat. Med. 1:27-31.

14. Hanahan, D., and Folkman, J. 1996. Patterns and emerging mechanisms of the angiogenic switch during tumorigenesis. Cell. 86:353-364.

15. Risau, W. 1997. Mechanisms of angiogenesis. Nature. 386:671-674

16. Denekamp, J. 1993. Review article: angiogenesis, neovascular proliferation and vascular pathophysiology as targets for cancer therapy. Br. J. Radiol. 66:181-196.

17. Chaplin, D.J., Pettit, G.R., Parkins, C.S., and Hill, S.A. 1996. Antivascular approaches to solid tumour therapy: evaluation of tubulin binding agents. Br.J. Cancer. 27:S86-S88.

18. Chaplin, D.J., Pettit, G.R., and Hill, S.A. 1999. Antivascular approaches to solid tumour therapy: evaluation of combretastatin A4 phosphate. Anticancer Res. 19:189-195.

19. Chabner, B.A., and Horwitz, S.B. 1990. Plant alkaloids. Cancer Chemother. Biol. Response Modif. 11:74-81.

20. McGown, A.T., and Fox, B.W. 1989. Structural and biochemical comparison of the anti-mitotic agents colchicine, combretastatin A4 and amphethinile. Anticancer Drug Des. 3:249-254.

21. Pettit, G.R., et al. 1989. Isolation and structure of the strong cell growth and tubulin inhibitor combretastatin A-4. Experientia. 45:209-211.

22. Dark, G.G., et al. 1997. Combretastatin A-4, an agent that displays potent and selective toxicity toward tumor vasculature. Cancer Res. 57:1829-1834.

23. Iyer, S., et al. 1998. Induction of apoptosis in proliferating human endothelial cells by the tumor-specific antiangiogenesis agent combretastatin A-4. Cancer Res. 58:4510-4514.

24. Grosios, K., Holwell, S.E., McGown, A.T., Pettit, G.R., and Bibby, M.C. 1999. In vivo and in vitro evaluation of combretastatin A-4 and its sodium phosphate prodrug. Br. J. Cancer. 81:1318-1327.

25. Galbraith, S.M., et al. 2001. Effects of combretastatin A4 phosphate on endothelial cell morphology in vitro and relationship to tumour vascular targeting activity in vivo. Anticancer Res. 21:93-102.

26. Esser, S., Lampugnani, M.G., Corada, M., Dejana, E., and Risau, W. 1998. Vascular endothelial growth factor induces VE-cadherin tyrosine phosphorylation in endothelial cells. J. Cell Sci. 111:1853-1865.

27. Zhang, F., et al. 2004. Adenovirus E4 gene promotes selective endothelial cell survival and angiogenesis via activation of the vascular endothelial-cadherin/Akt signaling pathway. J. Biol. Chem. 279:11760-11766.

28. Orford, K., Crockett, C., Jensen, J.P., Weissman, A.M., and Byers, S.W. 1997. Serine phosphorylation-regulated ubiquitination and degradation of beta-catenin. J. Biol. Chem. 272:24735-24738.

29. Liu, C., et al. 1999. beta-Trcp couples beta-catenin phosphorylation-degradation and regulates Xenopus axis formation. Proc. Natl. Acad. Sci. U. S. A. 96:6273-6278.

30. May, C., et al. 2005. Identification of a transiently exposed VE-cadherin epitope that allows for specific targeting of an antibody to the tumor neovasculature. Blood. 105:4337-4344.

31. Kermani, P., et al. 2005. Neurotrophins promote revascularization by local recruitment of $\mathrm{TrkB}^{+}$ endothelial cells and systemic mobilization of hematopoietic progenitors. J. Clin. Invest. 115:653-663. doi:10.1172/JCI200522655.

32. Grossmann, J. 2002. Molecular mechanisms of "detachment-induced apoptosis-Anoikis". Apoptosis. 7:247-260.

33. Weis, S., et al. 2004. Src blockade stabilizes a Flk/ cadherin complex, reducing edema and tissue injury following myocardial infarction. J. Clin. Invest. 113:885-894. doi:10.1172/JCI200420702.

34. Jain, R.K. 2003. Molecular regulation of vessel maturation. Nat. Med. 9:685-693.

35. Winkler, F., et al. 2004. Kinetics of vascular normalization by VEGFR2 blockade governs brain tumor response to radiation: role of oxygenation, angiopoietin-1, and matrix metalloproteinases. Cancer Cell. 6:553-563. 